Jumping Through the Transfinite: The Master Code Hierarchy of Turing Degrees Author(s): Harold T. Hodes

Source: The Journal of Symbolic Logic, Jun., 1980, Vol. 45, No. 2 (Jun., 1980), pp. 204220

Published by: Association for Symbolic Logic

Stable URL: https://www.jstor.org/stable/2273183

JSTOR is a not-for-profit service that helps scholars, researchers, and students discover, use, and build upon a wide range of content in a trusted digital archive. We use information technology and tools to increase productivity and facilitate new forms of scholarship. For more information about JSTOR, please contact support@jstor.org.

Your use of the JSTOR archive indicates your acceptance of the Terms \& Conditions of Use, available at https://about.jstor.org/terms 


\title{
JUMPING THROUGH THE TRANSFINITE: THE MASTER CODE HIERARCHY OF TURING DEGREES ${ }^{1}$
}

\author{
HAROLD T. HODES
}

\begin{abstract}
Where $\underline{a}$ is a Turing degree and $\xi$ is an ordinal $<\left(\aleph_{1}\right)^{L q}$, the result of performing $\xi$ jumps on $\underline{a}, \underline{a}^{(\xi)}$, is defined set-theoretically, using Jensen's fine-structure results. This operation appears to be the natural extension through $\left(\aleph_{1}\right)^{L e}$ of the ordinary jump operations. We describe this operation in more degree-theoretic terms, examine how much of it could be defined in degree-theoretic terms and compare it to the single jump operation.
\end{abstract}

1. Basic definitions and results. For $A \leq \omega$, let:

$$
\begin{aligned}
L_{0}[A]=M_{0}[A]=\{x \mid x \text { is hereditarily finite }\} & \\
L_{\alpha+1}[A] & =\left\{x \mid x \text { is first-order definable over }\left\langle L_{\alpha}[A] ; \in \uparrow L_{\alpha}[A], A ; L_{\alpha}[A]\right\rangle\right\} ; \\
L_{\lambda}[A] & =\bigcup L_{\alpha}[A] ; \\
M_{\omega \alpha+n}[A] & =\Delta_{n}\left(\left\langle L_{\alpha}[A] ; \in \uparrow L_{\alpha}[A], A ; L_{\alpha}[A]\right\rangle\right) \text { for } n \geq 1 ; \\
M_{\omega \alpha}[A] & =L_{\alpha}[A] .
\end{aligned}
$$

Clearly $M_{\omega(\alpha+1)}[A]-M_{\omega \alpha}[A]=L_{\alpha+1}[A]-L_{\alpha}[A] .\left\langle M_{\alpha}[A]\right\rangle_{\alpha}$ is introduced only for perspicacious statement of results. All proofs will use $\left\langle L_{\alpha}[A]\right\rangle_{\alpha}$. Note that if $A \equiv{ }_{\Gamma} B$ then $M_{\alpha}[A]=M_{\alpha}[B]$. Thus for a Turing degree $\underline{a}$, we may define $M_{\alpha}^{\underline{\alpha}}=$ $M_{\alpha}[A]$ and $L_{\bar{\alpha}}^{a}=L_{\alpha}[A]$, for $A \in \underline{a}$. We let $M_{\alpha}[\varnothing]=M_{\bar{\alpha}}^{0}=M_{\alpha}$ and $L_{\alpha}[\varnothing]=$ $L_{\bar{\alpha}}^{0}=L_{\alpha}$. All of the following definitions are given for $\underline{a}=\underline{0}$. They relativize to arbitrary $\underline{a}$ in the obvious way. As usual, $L_{\bar{\alpha}}^{0}=L_{\alpha}, M_{\bar{\alpha}}^{0}=M_{\alpha}$. Unless otherwise indicated, lower case Greek letters range over $\left(\kappa_{1}\right)^{L} ; \lambda$ always ranges over limit ordinals.

$[\alpha, \beta)=\{\gamma \mid \alpha \leq \gamma<\beta\} .[\alpha, \beta)$ is an $M$-gap iff $\left(M_{\beta}-M_{\alpha}\right) \cap \omega^{\omega}=\varnothing ;[\alpha, \beta)$ is an $M$-gap iff $\left(L_{\beta}-L_{\alpha}\right) \cap \omega^{\omega}=\varnothing . \alpha$ is an $M$-gap ordinal iff $[\alpha, \alpha+1)$ is an $M$-gap; $\alpha$ is an $L$-gap ordinal iff $[\alpha, \alpha+1)$ is an $L$-gap. $\alpha$ is an $M$-index iff $\alpha$ is not an $M$-gap ordinal; $\alpha$ is an $L$-index iff $\alpha$ is not an $L$-gap ordinal. $\alpha$ starts an $M$-gap iff $\alpha$ is an $M$-gap ordianl and is the supremum of $M$-indices; $\alpha$ starts an $L$-gap iff $\alpha$ is an $L$-gap ordinal and is the supremum of $L$-indices. Let $F(\alpha)$ be the maximum $\beta$ such that $[\alpha, \alpha+\beta)$ is an $M$-gap. Thus $\alpha$ is an $M$-gap ordinal iff $F(\alpha) \neq$ 0 . If $\alpha$ starts an $M$-gap, $F(\alpha)$ is the length of that gap.

Let Ind: $\left(\boldsymbol{N}_{1}\right)^{L} \rightarrow\left(\boldsymbol{N}_{1}\right)^{L}$ enumerate the $M$-indices in increasing order. Clearly

Received September 8, 1977.

${ }^{1}$ Thanks to the referee for finding several major and many minor errors. Special thanks to $\mathrm{F}$. Abramson for suggesting the use of modified Steel conditions in the proofs of Lemmas 1 and 2 under Case 3. Writing of this paper was in part supported by a Fellowship from the Mellon Foundation. 
$\alpha \leq \operatorname{Ind}(\alpha)$. If $\alpha<\operatorname{Ind}(\alpha)$, it is because Ind was temporarily "thrown off" by an $M$-gap. $A \subseteq \omega$ is a master code for $\alpha$ iff $M_{\alpha+1} \cap 2^{\omega}=\left\{B \subseteq \omega \mid B \leq_{\mathrm{T}} A\right\}$. Clearly this notion is invariant under Turing equivalence. Thus a Turing degree $\underline{b}$ is a master code for $\xi$ iff $\underline{b}$ is the degree of a master code for $\xi$.

The Fundamental TheOREM. $\xi$ is an $M$-index iff there is a master code for $\xi$; furthermore, if $\underline{b}$ is the master code for $\xi$ then $\underline{b}^{\prime}$ is the master code for $\xi+1$.

We are now ready to extend the jump operation through $\left(\kappa_{1}\right)^{L}$. Let $\underline{0}^{(\xi)}=$ the master code for Ind $(\xi)$. The previous definitions and the Fundamental Theorem relativize to an arbitrary degree $\underline{a}$. Thus we may define $\underline{a}^{(\xi)}=$ the $\underline{a}$-master code for $\operatorname{Ind}^{a}(\xi)$, for $\xi<\left(\boldsymbol{\kappa}_{1}\right)^{L \underline{a}}$.

The central results of this paper characterize the function $\xi \mapsto \underline{0}^{(\xi)}$ in more degree-theoretic terms. We now introduce the machinery needed to state these results.

$\operatorname{Ind}(\alpha) \neq \alpha$ iff $(\exists \beta)(\beta$ starts an $M$-gap and $\beta \leq \alpha<\beta+F(\beta) \cdot \omega)$. Let $J(\alpha)=$ the least strict upper-bound on $\{\operatorname{Ind}(\xi) \mid \xi<\alpha\} . \alpha \leq J(\alpha)$. In fact, $J(\alpha)>\alpha$ iff $\operatorname{Ind}(\alpha)>\alpha$ and $\alpha$ does not start an $M$-gap. $J(\alpha) \neq \operatorname{Ind}(\alpha)$ iff $\alpha$ starts an $M$-gap. $\operatorname{Ind}(\alpha)=J(\alpha)+F(J(\alpha))$.

We divide limit ordinals below $\left(\aleph_{1}\right)^{L}$ into three cases.

Case 1. $J(\lambda)$ is not a limit of $M$-gaps.

Case 2. $J(\lambda)$ is a limit of $M$-gaps and $F(J(\lambda))<\omega$.

Case 3. Otherwise.

Notice that $F(J(\lambda)) \geq \omega$ iff $\lambda$ falls under Case 3 .

In subsequent proofs, further subdivision is needed.

Case 1.1. $\lambda$ falls under Case 1 and $J(\lambda)$ is an $M$-index.

Case 1.2. $\lambda$ falls under Case 1 but not Case 1.1.

$J(\lambda)$ is an $M$-gap ordinal iff $J(\lambda)$ is admissible iff $\lambda$ is admissible and locally countable. Notice that if $\lambda$ is not under Case 1.1, then $J(\lambda)=\lambda=\omega \lambda$. For $\lambda$ under Case $1, \lambda$ falls under Case 1.1 iff $F(J(\lambda))=0$, and $\lambda$ falls under Case 1.2 iff $F(J(\lambda))$ $=F(\lambda)=1$. The least Case 1.1 ordinal is $\omega$, and $\operatorname{Ind}(\omega)=\omega$. The least Case 1.2 ordinal is $\omega_{1}^{C K}=\omega_{1}$ and $\operatorname{Ind}\left(\omega_{1}\right)=\omega_{1}+1$. The least Case 2 ordinal is $\sup \left\{\omega_{n}^{C K} \mid n<\omega\right\}$ $=\omega_{\omega}$, and $\operatorname{Ind}\left(\omega_{\omega}\right)=\omega_{\omega}$. The least Case 3 ordinal is $\beta_{0}$, and $\operatorname{Ind}\left(\beta_{0}\right)=\beta_{0}+\omega$.

Between terms denoting Turing degrees, " $\leq$ " represents Turing reducibility. A set $I$ of Turing degrees is an ideal iff it is closed under join and downward-closed under $\leq$. If $I$ is an ideal, the pair $(\underline{b}, \underline{c})$ is $I$-exact iff for any $\underline{a}, \underline{a} \in I$ iff $\underline{a} \leq \underline{b}$ and $\underline{a} \leq \underline{c}$. If $(\underline{b}, \underline{c})$ is $I$-exact we shall also call $(\underline{b} \vee \underline{c}) I$-exact. Let $I_{\lambda}$ be the minimal ideal containing $\left\{\underline{0}^{(\xi)} \mid \xi<\lambda\right\}$. By definitions, $\bigcup I_{\lambda}=M_{J(\lambda)} \cap \omega^{\omega}=L_{\gamma} \cap \omega^{\omega}=$ $L_{\beta} \cap \omega^{\omega}$ where $\omega \cdot \gamma=J(\lambda)$ and $\operatorname{Ind}(\lambda)=\omega \cdot \beta+n$ for $n<\omega$.

The following results extend the characterization of $\left(\lambda \xi \cdot \underline{0}^{(\xi)}\right) \uparrow \beta_{0}$ provided in [6]. Let $\mu_{\lambda}=$ the least $\mu$ such that $\left\{\underline{a}^{(\mu)} \mid \underline{a}\right.$ is $I_{\lambda}$-exact $\}$ has a least member.

THEOREM 1. $\mu_{\lambda}$ exists. In fact,

$$
\mu_{\lambda}= \begin{cases}2+F(J(\lambda)) & \text { for } \lambda \text { under Case } 1, \\ 3+F(J(\lambda)) & \text { for } \lambda \text { under Case } 2 \text { or Case } 3 .\end{cases}
$$

Thus $\mu_{\lambda}=3+F(\lambda)$ for $\lambda$ under Cases 2 or 3 , and $\mu_{\lambda}=F(\lambda)$ in Case 3 . 
THEOREM 2. $\underline{0}^{(\lambda)}$ is the least member of $\left\{\underline{a}^{\left(\mu_{\lambda}\right)} \mid \underline{a}\right.$ is $I_{\lambda}$-exact $\}$. Moreover, it is the least member of $\left\{\underline{a}^{\left(\mu_{\lambda}\right)} \mid \underline{a}\right.$ is $I_{\lambda}$-exact and $\operatorname{Ind}(\lambda)$ is recursive in $\left.\underline{a}\right\}$.

Where $U$ is a predicate of Turing degrees, $\underline{a}$ is a $\xi$-low $U$ iff $\underline{a}$ is a $U$ and for any $\underline{b} \in U, \underline{a} \leq \underline{b}^{(\xi)}$.

THEOREM 3. For $\xi<\mu_{\lambda}$, there is no $\xi$-low $I_{\lambda}$-exact degree.

These theorems shall all be derived from the following lemmas. Let

$$
G(\lambda)= \begin{cases}2+F(J(\lambda)) & \text { if } \lambda \text { falls under Case } 1, \\ 3+F(J(\lambda)) & \text { if } \lambda \text { falls under Case } 2 \text { or Case } 3 .\end{cases}
$$

LEMMA 1. There is an $I_{\lambda}$-exact pair $(\underline{b}, \underline{c})$ such that $(\underline{b} \vee \underline{c})^{(G(\lambda))} \leq \underline{0}^{(\lambda)}$ and $\operatorname{Ind}(\lambda)$ is recursive in $\underline{b}$ and in $\underline{c}$.

LEMMA 2. For any $\underline{d} \notin I_{\lambda}$ there is an $I_{\lambda}$-exact pair $(\underline{b}, \underline{c})$ such that for any $\xi<G(\lambda)$, $\underline{d} \ddagger(\underline{b} \vee \underline{c})^{(\xi)}$ and $\operatorname{Ind}(\lambda)$ is recursive in $\underline{b}$ and in $\underline{c}$.

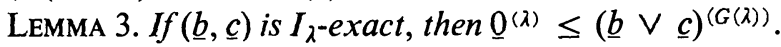

By Lemmas 1 and $3, \underline{0}^{(\lambda)}$ is the least member of $\left\{\underline{a}^{(G(\lambda))} \mid \underline{a}\right.$ is $I_{\lambda}$-exact $\}$ and of $\left\{\underline{a}^{(G(\lambda))} \mid \underline{a}\right.$ is $I_{\lambda}$-exact and $\left.\operatorname{Ind}(\lambda)<\omega_{1}^{(b \vee c)}\right\}$. Thus $\mu_{\lambda}$ exists. By definition of $\mu_{\lambda}$, $\left\{\underline{a}^{\left(\mu_{\lambda}\right)} \mid \underline{a}\right.$ is $I_{\lambda}$-exact $\}$ has a least member $\underline{d}$. Since $\underline{d} \notin I_{\lambda}$, by Lemma 2 if $\mu_{\lambda}<G(\lambda)$, $\underline{d}$ is not least. Thus $\mu_{\lambda}=G(\lambda)$. Lemma 2 easily proves Theorem 3 .

COROLlaRy. $\underline{0}^{(\lambda)}$ is the least member of $\left\{\underline{a}^{\left(-1+\mu_{\lambda}\right)} \mid \underline{a}\right.$ is a u.u.b.on $\left.I_{\lambda}\right\}$ and of $\left\{\underline{a}^{\left(\mu_{\lambda}\right)} \mid\right.$ $\underline{a}$ is a weak u.u.b. on $\left.I_{\lambda}\right\}$. For $\xi<\left(-1+\mu_{\lambda}\right)$ there is no $\xi$-low u.u.b. on $I_{\lambda} ;$ for $\xi<\mu_{\lambda}$ there is no $\xi$-low weak u.u.b. on $I_{\lambda}$.

(See [4] for the definition of a u.u.b. and a weak u.u.b.) This corollary connects these results with the apparatus of [2].

We state, mostly without proof, some basic facts about gaps.

1. If $\alpha$ starts an $M$-gap or an $L$-gap, $\alpha$ is a limit ordinal and $\omega \alpha=\alpha$.

2. If $\alpha$ starts an $L$-gap, then $\alpha$ starts an $M$-gap.

3. If $\alpha$ is a supremum of $L$-indices, $L_{\alpha} \vDash V=H C$ (i.e. "everything is countable").

4. If $\alpha$ starts an $M$-gap, $\alpha$ is the supremum of $L$-indices.

A $\Delta_{n}$ comprehension axiom is a sentence of the form:

$$
(\forall x \in \omega)(\phi x \leftrightarrow \phi x) \rightarrow(\exists y)(y \subseteq \omega \&(\forall x)(x \in y \leftrightarrow \phi x)) .
$$

where $\phi$ is $\Sigma_{n}$ and $\phi$ is $\Pi_{n} . \Delta_{n}$ CA is the set of $\Delta_{n}$ comprehension axioms.

5. If $F(\omega \alpha) \geq n$ then $L_{\alpha} \vDash \Delta_{n}$ CA.

6. If $\alpha$ starts an $M$-gap then $\alpha$ starts an $L$-gap iff $F(\alpha) \geq \omega$.

7. If $\alpha$ starts an $M$-gap and $F(\alpha) \geq n$ then $\alpha$ is $\Sigma_{n}$-admissible.

Proof. Use Jensen's result on the $\Sigma_{n}$ uniformizability of $L_{\alpha}$.

8. $\alpha$ starts an $L$-gap iff $L_{\alpha} \vDash \mathrm{ZF}^{-}+V=H C$; if $\alpha$ starts an $L$-gap, $L_{\alpha} \cap \omega^{\omega}$ is a $\beta$-model of analysis. $\left(\mathrm{ZF}^{-}=\mathrm{ZF}-\right.$ - Power Set $\}$.) See [8].

9. $\lambda$ is a limit of $M$-gaps iff $M_{\lambda}$ is closed under hyperjump iff $M_{\lambda} \cap \omega^{\omega}$ is $\Pi_{1}^{1}$ absolute.

We freely identify binary relations on $\omega$ with subsets of $\omega$ via the coding scheme $n=\left\langle(n)_{0},\left(n_{1}\right)\right\rangle$. Thus for $X \subseteq \omega$, structures $\langle X, R, A\rangle, R \subseteq X^{2}, A \subseteq X$, may be 
identified with reals. An arithmetic copy of $\left\langle L_{\alpha}[A] ; \in \uparrow\left(L_{\alpha}\left[A^{2}\right]\right), A\right\rangle$ hereafter called an arithmetic copy of $L_{\alpha}[A]$, is a structure $\langle X, R, A\rangle, X \subseteq \omega$, isomorphic to $\left\langle L_{\alpha}[A] ; \in \uparrow\left(L_{\alpha}[a]\right)^{2}, A\right\rangle$, coded as single real. Hereafter $E_{\alpha}[A]$ ranges over arithmetic copies of $L_{\alpha}[A]$. Let $\operatorname{Th}_{n}(\langle X ; R, A\rangle)$ be $\operatorname{Th}(\langle X ; R, A ; X\rangle) \cap\left(\Sigma_{n} \cup I_{n}\right)$, the $n$ quantifier theory of $\langle X ; R, A ; X\rangle$, with each member of $X$ viewed as a name of itself. For $X \subseteq \omega, \mathrm{Th}_{n}(\langle X ; R, A\rangle)$ may be viewed as a single real. The following standard facts about the arithmetic hierarchy provide motivation for this paper: if $E_{0}[A] \leq_{\mathrm{T}} A$, then $\mathrm{Th}_{n}\left(E_{0}[A]\right) \equiv_{\mathrm{T}} A^{(n)}$, and there is an $E_{1}[A]$ canonically constructed from $E_{0}[A]$ such that $\operatorname{Th}\left(E_{0}[A]\right) \equiv_{\mathrm{T}} \operatorname{Th}_{0}\left(E_{1}[A]\right) \equiv_{\mathrm{T}} A^{(\omega)}$.

The Fundamental Theorem is proved in [6]. The proof makes use of Jensen's $\Sigma_{n}$ uniformization theorem, transferred from the $J$ to the $L$ hierarchy. The proof of that uses Jensen's notion of a $\Sigma_{n}$ master code for an arbitrary $L_{\alpha}$. It might seem more direct to imitate Jensen's proof, which proves $\Sigma_{n}$ uniformization and the existence of $\Sigma_{n}$ master codes simultaneously, with $\Delta_{n}$ uniformization and $\Delta_{n}$ master codes, thereby avoiding mention of $\Sigma_{n}$ master codes. But this seems to be impossible.

The proof of the Fundamental Theorem proceeds by proving the following fact, which we shall misleadingly call a corollary.

If $L_{\alpha} \not \neq \Delta_{n+1}$ CA then $\Delta_{n+1}\left(L_{\alpha}\right)$ contains a real of the form $\operatorname{Th}_{n}\left(E_{\alpha}\right)$. Thus the master code for $\omega \alpha+n$ is the least degree of the form $\operatorname{deg}\left(\operatorname{Th}_{n}\left(E_{\alpha}\right)\right)$.

The ordinary jump on $\mathscr{D}$ corresponds to a canonical jump function * on $P \omega$ : $\operatorname{deg}(A)^{\prime}=\operatorname{deg}\left(A^{*}\right)$. Unfortunately, an arbitrary transfinite jump on $\mathscr{D}$ seems to be associated with no canonical such function on $P \omega$.

§2. Proofs of Lemmas 1, 2 and 3. Lemmas 1 and 2 for $\lambda$ under Cases 1 or 2 are proved in [6]. For the sake of a complete presentation we sketch those proofs here.

Suppose $\lambda$ falls under Cases 1 or 2 . Let $n=F(J(\lambda))$; let $J(\lambda)=\omega \alpha$. Thus for some $E_{\alpha}, \underline{0}^{(\lambda)}=\operatorname{deg}\left(\operatorname{Th}_{n}\left(E_{\alpha}\right)\right)$. To prove Lemma 1 it suffices to construct $B$ and $C \in 2^{\omega}$ such that

(1) $(B, C)$ is exact for $L_{\alpha} \cap \omega^{\omega}$;

(2.1) if $\lambda$ falls under Case 1 then

$$
(B \oplus C)^{(2+n)} \in A_{n+1}\left(L_{\alpha}\right) ;
$$

(2.2) if $\lambda$ falls under Case 2 then

$$
(B \oplus C)^{(3+n)} \in \Delta_{n+1}\left(L_{\alpha}\right) .
$$

To prove Lemma 2 it suffices, given $\underline{d} \notin I_{\lambda}$ and $f \in \underline{d}$, to construct $B$ and $C \in 2^{\omega}$ such that (1) is true and

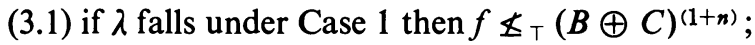

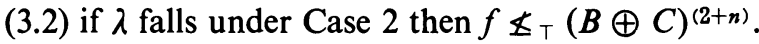

We now prove Lemmas 1 and 2, using forcing with uniformly recursively pointed perfect trees in an arithmetic setting. Fix a forcing language built from number variables, numerals, predicate constants for primitive recursive predicates on $2^{\omega} \times$ $2^{\omega} \times \omega$, and generic predicate constants $\underline{B}$ and $\underline{C}$. Build prenex sentences from $\exists$ and $\neg$, with the usual $\Pi_{i}^{0}, \Sigma_{i}^{0}$ classification. Conditions are as in [6]: pairs $(P, Q)$ where $P$ and $Q$ are uniformly recursively pointed perfect trees from $L_{\alpha} \cap \omega^{\omega}$ and 
$P \equiv{ }_{\mathrm{T}} Q \cdot(P, Q)$ extends $(R, S)$ iff $P$ and $Q$ are subtrees of $R$ and $S$ respectively. $[P]$ is the set of characteristic functions, identified with members of $P \omega$, which lie along branches of $P .[P, Q]=[P] \times[Q] .(P, Q) H \varnothing$ iff for any $(B, C) \in[P, Q]$, $(B, C) \vDash \varnothing$, where $\varnothing \in \Pi_{i \leq 2}^{0}$. The other clauses are standard:

$$
\begin{gathered}
(P, Q) H(\exists x) \varnothing \text { iff for some } n<\omega(P, Q) H \varnothing(x / n) ; \\
(P, Q) H \neg \varnothing \text { iff for every }(R, S) \text { extending }(P, Q), \\
(R, S) H \varnothing, \text { for } \varnothing \in \Sigma_{i>2}^{0} .
\end{gathered}
$$

By Lemma 3.5 of [6], some condition extending $(P, Q)$ decides $\varnothing$.

We must compute the definitional complexity over $L_{\alpha}$ of forcing restricted to $\left(\Sigma_{G(\lambda)}^{0} \cup \Pi_{G(\lambda)}^{0}\right)$. The class of conditions and the extends relation are $\Sigma_{3}^{0}$ and $\Pi_{2}^{0}$ respectively. By Lemma 3.8 of [6], forcing restricted to $\left(\Sigma_{0}^{2} \cup \Pi_{2}^{0}\right)$ is $\Sigma_{3}^{0}$. So if $\lambda$ falls under Case 1.1, forcing restricted to $\left(\Sigma_{G(\lambda)} \cup \Pi_{G(\lambda)}\right)$ is $\Delta_{1}$ over $L_{\alpha}$. Forcing restricted to $\left(\Sigma_{3} \cup \Pi_{3}\right)$ is $\Pi_{1}^{1}$ over $L_{\alpha} \cap \omega^{\omega}$, so clearly $\Delta_{2}$ over $L_{\alpha}$. So for $\lambda$ under Case 1 , forcing for $\left(\Sigma_{G(\lambda)} \cup \Pi_{G(\lambda)}\right)$ is $\Delta_{F(J(\lambda))+1}$ over $\left.L_{\alpha}\right)$.

Suppose $\lambda$ falls under Case 2. $L_{\alpha} \cap \omega^{\omega}$ is $\Pi_{1}^{1}$ absolute. So forcing restricted to $\left(\Sigma_{3}^{0} \cup \Pi_{3}^{0}\right)$ is $\Pi_{1}$ over $L_{\alpha}$. But by the Kleene basis theorem we can show that it is also $\Sigma_{1}$ over $L_{\alpha}$; suppose $(P, Q) H \varnothing$ if and only if $\left(\forall f \in \omega^{\omega}\right) R(f, P, Q, \varnothing)$, where $R \in \Sigma_{1}^{0}$, $W^{X}$ is the hyperjump of $X$. Then $\left(\forall f \in \omega^{\omega}\right) R(f, P, Q, \varnothing)$ iff $\left(\forall f \leq_{\mathrm{T}} W^{(P \oplus Q)}\right)$ $\cdot R(f, P, Q, \varnothing)$ iff $L_{\alpha} H(\exists \xi)$ ( $\xi$ admissible \& $(P, Q) \in L_{\xi} \&\left(\forall f \in L_{\xi+1} \cap \omega^{\omega}\right)$ $\cdot R(f, P, Q, \varnothing))$. So in Case 2, following up the definition of forcing, forcing for $\left(\Sigma_{i+3}^{0} \cup \Pi_{i+3}^{0}\right)$ is $\Delta_{i+1}$ over $L_{\alpha}$, thus for $\left(\Sigma_{G(\lambda)}^{0} \cup \Pi_{G(\lambda)}^{0}\right)$ is $\Delta_{F(J(\lambda))+1}$ over $L_{\alpha}$.

Let $\left\langle\varnothing_{i}\right\rangle_{i \in \omega}$ and $\left\langle A_{i}\right\rangle_{i \in \omega}$ be $\Delta_{n+1}\left(L_{\alpha}\right)$ enumerations of $\left(\Sigma_{G(\lambda)} \cup \Pi_{G(\lambda)}\right)$ and $L_{\alpha} \cap$ $2^{\omega}$ respectively. The latter exists by the corollary to the Fundamental Theorem. Let

$$
\begin{gathered}
P_{0}=Q_{0}=\mathrm{id}, \quad\left(P_{2 i+1}, Q_{2 i+1}\right)=\left(P_{2 i} * A_{i}, Q_{2 i} * A_{i}\right) ; \\
\left(P_{2 i+2}, Q_{2 i+2}\right)=\text { the }<_{L} \text { least extension of }\left(P_{2 i+1}, Q_{2 i+1}\right) \text { deciding } \varnothing_{i},
\end{gathered}
$$

where $P * A$ is the canonical result of coding $A$ into $P$; see [6, Lemma 3.3]. $\left\langle\left(P_{i}, Q_{i}\right)\right\rangle_{i \in \omega} \in \Delta_{n+1}\left(L_{\alpha}\right)$. Let $(B, C)=\bigcap_{i<\omega}\left(P_{i}, Q_{i}\right]$. The usual forcing $=$ truth lemma states that $(B, C) H \varnothing_{i}$ iff $\left(P_{2 i+1}, Q_{2 i+1}\right) H \varnothing_{i}$. (1) follows easily from the odd steps and Lemma 3.7 of [6]. Since $(B \oplus C)^{(G(\lambda))}$ is defined by a $\Sigma_{G(\lambda)}$ formula, (2.1) and (2.2) are satisfied.

To prove Lemma 2 it shall be necessary to prove the following.

Sublemma 1. For any $i$ and any condition $(P, Q)$ there is an $m$ and a condition $(R, S)$ extending $(P, Q)$ such that either

$$
(R, S) H^{\text {“ }} \neg\{\underline{i}\}^{(\underline{B} \oplus \underline{C})(G(\lambda)-1)}(\underline{m}) \text { converges" }
$$

or

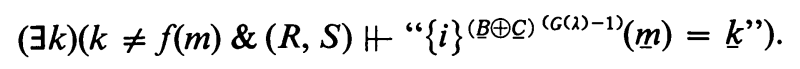

Suppose $(P, Q)$ and $i$ are a counterexample, i.e. for any $m$ and any $(R, S)$ extending $(P, Q)$ : 


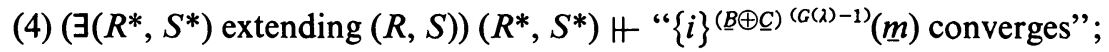
and

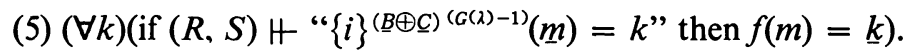

Thus $f(m)=k$ iff

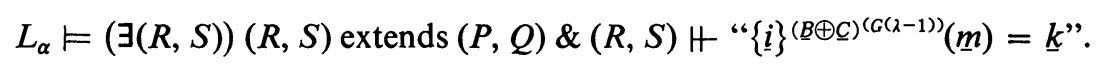

By the previous results on the definitional complexity of forcing, the above definition is $\Sigma_{n}$. Thus, because $f$ is a function, $f \in \Delta_{n}\left(L_{\alpha}\right)$. But $L_{\alpha} \vDash \Delta_{n}$ CA. So $f \in$ $L_{\alpha}$, contrary to choice of $f$.

We now finish the proof of Lemma 2. Let $\left\langle\varnothing_{i}\right\rangle_{i \in \omega}$ and $\left\langle A_{i}\right\rangle_{i \in \omega}$ be enumerations of $\left(\Sigma_{G(\lambda)-1} \cup \Pi_{G(\lambda)-1}\right)$ and $L_{\alpha} \cap 2^{\omega}$ respectively; let

$$
\begin{aligned}
& P_{0}=Q_{0}=\mathrm{id} \\
& \left(P_{3 i+1}, Q_{3 i+1}\right)=\left(P_{3 i} * A_{i}, Q_{3 i} * A_{i}\right) \text {; } \\
& \left(P_{3 i+2}, Q_{3 i+2}\right)=\text { an extension of }\left(P_{3 i+1}, Q_{3 i+1}\right) \text { deciding } \varnothing \text {; } \\
& \left(P_{3 i+3}, Q_{3 i+3}\right)=\text { an extension of }\left(P_{3 i+2}, Q_{3 i+2}\right) \text { such that for some } m \text {, } \\
& \text { either }
\end{aligned}
$$

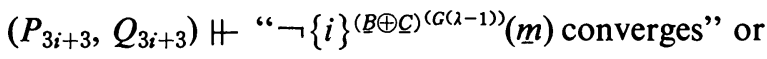

$$
\begin{aligned}
& \text { for some } k \neq f(m) \text {, }
\end{aligned}
$$

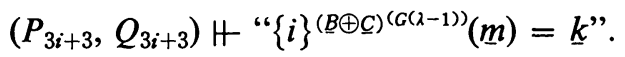

Let $(B, C)=\bigcap_{i<\omega}\left[P_{i}, Q_{i}\right] .(1)$ is immediate as in Lemma 1. Stages of the form $3 i$ insure the truth of (3.1) and (3.2). Note that if $f \in \Delta_{n+1}\left(L_{\alpha}\right)$ the above construction can be made $\Delta_{n+1}$ over $L_{\alpha}$. So for $\underline{d} \leq \underline{0}^{(\lambda)}$ we could choose $(\underline{b}, \underline{c})$ so that $\underline{d} \not$ $(\underline{b} \vee \underline{c})^{(G(\lambda)-1)}$ and $(\underline{b} \vee \underline{c})^{(G(\lambda))} \leq \underline{0}^{(\lambda)}$, so $(\underline{b} \vee \underline{c})^{(G(\lambda))}=\underline{0}^{(\lambda)}$ by Lemma 3 .

Suppose that $\lambda$ falls under Case 3. Recall that $J(\lambda)=\lambda=\omega \lambda$. Let $F(\lambda)=\omega \beta+$ $n$. For some $E_{\lambda+\beta}, \underline{0}^{(\lambda)}=\operatorname{deg}\left(\operatorname{Th}_{n}\left(E_{\lambda+\beta}\right)\right)$. To prove Lemma 1 it suffices to construct $B$ and $C \in 2^{\omega}$ such that (1) is true and

(8) For some $E_{\beta}[B \oplus C], \mathrm{Th}_{n}\left(E_{\beta}[B \oplus C]\right) \in \Delta_{n+1}\left(L_{\lambda+\beta}\right)$;

(9) $\lambda+\beta<\omega_{1}^{B}$ and $\lambda+\beta<\omega_{1}^{C}$;

(9) implies that $\operatorname{Ind}(\lambda)<\omega_{1}^{(B \oplus C)}$. Its purpose is more than decorative. Let $\underline{b}=$

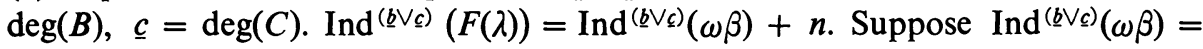
$\omega \gamma+m$. Then for some $E_{\gamma}[B \oplus C],(\underline{b} \vee \underline{c})^{(G(\lambda))}=\operatorname{deg}\left(\operatorname{Th}_{m+n}\left(E_{\gamma}[B \oplus C]\right)\right)$. If $\omega \beta+n<\omega \gamma+m$, we have no reason to expect that we can find $B, C$ and $E_{\gamma}[B \oplus C]$ such that $\operatorname{Th}_{m+n}\left(E_{\gamma}[B \oplus C]\right) \in \Delta_{n+1}\left(L_{\lambda+\beta}\right)$. However, (9) insures that Ind $^{(\underline{b} \vee c)}(\omega \beta)=\omega \beta$. Thus (8) suffices for Lemma 1. For Lemma 2, suppose that $\underline{d} \notin I_{\lambda}$ and $f \in \underline{d}$. If $\lambda$ falls under Case 3 it suffices to construct $B$ and $C \in 2^{\omega}$ such that (1) and (9) are true and

(10) $f \notin \Sigma_{n}\left(L_{\beta}[B \oplus C]\right)$.

As before, (9) insures that $(\underline{b} \vee \underline{c})^{(F(\lambda))}=\operatorname{deg}\left(\operatorname{Th}_{n}\left(E_{\beta}[B \oplus C]\right)\right)$ for some $E_{\beta}[B \oplus C]$. Furthermore, $\bigcup I_{B}^{(b \bigvee \underline{c})}=L_{\beta}[B \oplus C] \cap \omega^{\omega}$.

For both Lemmas 1 and 2 we shall obtain $B=B_{0} \oplus B_{1}$ and $C=C_{0} \oplus C_{1}$, such that $B_{0}$ and $C_{0}$ are Turing upper-bounds on $L_{\lambda} \cap \omega^{\omega}$ and such that $B_{1}$ and $C_{1}$ 
are wellfounded trees of height high enough to insure that $\omega_{1}^{B_{1}}$ and $\omega_{1}^{C_{1}}$ are greater than $\lambda+\beta$.

In Case 3, the proofs of Lemmas 1 and 2 use forcing for a ramified language. Fix a set $\boldsymbol{P}$ of one-place predicate constants. Let the lexicon of $\boldsymbol{L}_{\eta}^{*}[\boldsymbol{P}]$ consist of members of $\boldsymbol{P}, \neg, \&, \exists$, parentheses, countably many unranked variables, and for each $\xi<\eta$, countably many variables of rank $\xi$. Let the formation rules be as usual, except that $\ulcorner\underline{P}(v)\urcorner$ for $\underline{P} \in \boldsymbol{P}$ is well-formed iff $v$ has rank 0 . Call a formula with no bound unranked variables "ranked". Let $C_{0}[\boldsymbol{P}]$ be a set of standard names for members of $L_{0}$. Let $C_{\xi+1}[\boldsymbol{P}]$ be the set of terms $\hat{x}^{\xi} \phi\left(x_{1} / c_{1}, \ldots, x_{k} / c_{k}\right)$ such that $\phi$ is ranked with exactly the free variables $x^{\xi}, x_{1}, \ldots, x_{k}$, no bound variables of rank $>\xi, c_{1}, \ldots, c_{k} \in \bigcup_{\alpha \leq \xi} C_{\alpha}[\boldsymbol{P}]$. If $\boldsymbol{\xi}$ is a limit, let $C_{\xi}[\boldsymbol{P}]=\bigcup_{\alpha<\xi} C_{\alpha}[\boldsymbol{P}]$. Let $\boldsymbol{L}_{\eta}[\boldsymbol{P}]$ be the language which results by supplementing $L_{\eta}^{*}[P]$ by the constants in $C_{\eta}[P]$.

Identify terms and formulae of $L_{\eta}[P]$ with members of $L_{\eta}$ in some fixed way. The rank of term $c, \rho(c)$, is the least $\xi$ such that $c \in C_{\xi}[\boldsymbol{P}]$. A formula $\phi$ of $L_{\eta}[\boldsymbol{P}]$ is ranked iff it has no bound unranked variables. Its rank, $\rho(\phi)$, is the supremum of the ranks of its contained constants, predicate constants, and bound variables, where members of $\boldsymbol{P}$ have rank 1. Suppose $\boldsymbol{P}=\left\{\underline{\boldsymbol{P}}_{0}, \ldots, \underline{\boldsymbol{P}}_{k}\right\}$. For $i \leq k$, suppose $\underline{\boldsymbol{P}}_{i}$ is assigned to $P_{i} \subseteq \omega . L_{\eta}\left[P_{0}, \ldots, P_{k}\right]$ is defined in the obvious way, and obviously equals $L_{\eta}\left[P_{0} \oplus \cdots \oplus P_{k}\right] .\left\langle L_{\eta}\left[P_{0}, \ldots, P_{k}\right] ; \in \mid L_{\eta}\left[P_{0}, \ldots, P_{k}\right], P_{0}, \ldots, P_{k} ; L_{\eta}\left[P_{0}, \ldots, P_{k}\right]\right\rangle$ is the intended structure for $\boldsymbol{L}_{\eta}[\boldsymbol{P}]$. Note that for $\eta>0$, the intended structure contains each $P_{i}$ both as an extension of $\underline{P}_{i}$ and as an individual denoted by " $\hat{x}^{0}\left(P_{i}\left(x^{0}\right)\right)$ ". Variables of rank $\xi<\eta$ range over $L_{\xi}\left[P_{0}, \ldots, P_{k}\right] . c \in C_{\eta}[P]$ denotes a member of $L_{\rho(c)}\left[P_{0}, \ldots, P_{k}\right]$. Thus if $\phi$ is ranked, $\phi$ is interpretable over $L_{\rho(\phi)}\left[P_{0}, \ldots, P_{k}\right]$.

Let $\Pi_{0}=\Sigma_{0}=\left\{\phi \mid \phi\right.$ is ranked formula of $\left.\boldsymbol{L}_{\eta}[\boldsymbol{P}]\right\}$. Define $\Sigma_{n}$ and $\Pi_{n}$ as usual. For the proofs to follow, let $\boldsymbol{P}=\left\{\underline{B}_{0}, \underline{B}_{1}, \underline{C}_{0}, \underline{C}_{1}\right\}$ and let $L_{\beta}[\boldsymbol{P}]=L$; let $C_{\xi}[\boldsymbol{P}]=$ $C_{\xi}$.

To insure the truth of (9) we need conditions more complicated than those used up to now. Let $\delta$ be the maximum ordinal $\leq \lambda+\beta$ which is either admissible or a limit of admissibles.

A modified Steel condition is a finite function $z$ into $\delta$ such that $\operatorname{dom}(z) \subseteq$ Seq $-\{\langle\rangle\}, \operatorname{dom}(z)$ is closed under initial segments, and for $\sigma, \tau \in \operatorname{dom}(z)$, if $\sigma$ properly extends $\tau$ then $z(\sigma)<z(\tau)$. (Think of $\langle>$ as belonging to $\operatorname{dom}(z)$ and $z(\langle\rangle)=\delta$.) If $y$ and $z$ are such conditions, $z$ extends $y$ iff $z \uparrow \operatorname{dom}(y)=y$.

Let a condition be a quadruple $(P, Q, y, z)$, where $P$ and $Q$ are Turing equivalent uniformly recursively pointed perfect trees in $L_{\lambda}$ and $y$ and $z$ are modified Steel conditions. Understand "extends" componentwise. Hereafter, " $K$ " etc. shall range over conditions. Let the height of $K, \operatorname{ht}(K),=\max (\operatorname{range}(y) \cup \operatorname{range}(z))$. Let $\boldsymbol{K}=$ the set of conditions; $\boldsymbol{K}_{\xi}=\{K \mid \mathrm{ht}(K)<\xi\}$, where $\xi$ is a limit ordinal.

Let $<^{*}$ be the wellfounded relation on sentences of $L$ introduced by Cohen in his definition of forcing [3, p. 115]. Forcing for sentences in $L$ is defined by induction on $<^{*}$. Let $K=(P, Q, y, z)$.

$$
\begin{array}{ll}
K H \varnothing & \text { iff } \rho(\varnothing)=0 \text { and } \varnothing \text { is true; } \\
K H \underline{B}_{0}(\underline{k}) & \text { iff for every } X \in[P], k \in X ; \\
K H \underline{C}_{0}(\underline{k}) & \text { iff for every } X \in[Q], k \in X ;
\end{array}
$$


$K H \underline{B}_{1}(\underline{k}) \quad$ iff $k \in \operatorname{dom}(y)$ or $k=\langle\rangle$;

$K H \underline{C}_{1}(\underline{k}) \quad$ iff $k \in \operatorname{dom}(z)$ or $k=\langle\rangle$;

$K H-\varnothing \quad$ iff for every $K^{\prime}$ extending $K, K^{\prime} H+\varnothing$, for $\rho(-\varnothing)>0$;

$K H \varnothing \& \psi \quad$ iff $K H \varnothing$ and $K H \psi$ for $\rho(\varnothing \& \psi)>0$;

$K H\left(\exists x^{\xi}\right) \varnothing \quad$ iff for some $c \in C_{\xi}, K H \varnothing\left(x^{\xi} / c\right)$, for $\rho\left(\left(\exists x^{\xi}\right) \varnothing\right)>0$;

$K H(\exists x) \varnothing \quad$ iff for some $c \in C, K H \varnothing(x / c)$;

$K H c_{1} \in c_{2} \quad$ iff either (i) $\rho\left(c_{1}\right)<\rho\left(c_{2}\right)$ and $c_{2}$ is $\hat{x}^{\xi} \varnothing$ and

$K+\varnothing\left(x^{\xi} / c_{1}\right)$, or (ii) for some $c_{3}, \rho\left(c_{3}\right)<\rho\left(c_{2}\right)$

and $K H\left(\left(\forall x^{\xi}\right)\left(x^{\xi} \in c_{1} \leftrightarrow x^{\xi} \in c_{3}\right) \& c_{3} \in c_{2}\right)$

where $\rho\left(c_{1}\right)=\xi+1$, and in either case $\rho\left(c_{1} \in c_{2}\right)>0$.

Let $|\varnothing|$ be the ordinal for the position of $\varnothing$ in $<^{*} \uparrow\left\{\psi \mid \psi \in \Pi_{0}\right.$ and $\left.\rho(\psi)>0\right\}$. Thus sup $|\varnothing| \leq \omega \cdot(\lambda+\beta)$. In order to refer to $|\varnothing|$ in $L_{\lambda+\beta}$, code $\omega \cdot(\lambda+\beta)$ into $\omega \times(\lambda+\beta)$ in the canonical way; we shall freely identify $|\varnothing|$ with the appropriate member of $\omega \times(\lambda+\beta)$. For $\eta<\lambda+\beta$ and $\varnothing$ such that $\rho(\varnothing)>0$, $|\varnothing|<\omega \cdot \eta$ iff $\rho(\varnothing)<\eta$. From right to left this is clear; if $|\varnothing|<\omega \cdot \eta$, then for some $k<\omega$, $|\varnothing|=\omega \cdot \rho(\varnothing)+k$; so $\omega \cdot \rho(\varnothing)<\omega \eta$; so $\rho(\varnothing)<\eta$.

Sublemma 2. Forcing restricted to $\left(\Sigma_{n} \cup \Pi_{n}\right)$ sentences is $\Delta_{n+1}$ over $L_{\lambda+\beta}$.

Let $H$ be the characteristic function for forcing. We shall prove that $H$ i $\left(\boldsymbol{K} \times \Pi_{0}\right) \in \Sigma_{1}\left(L_{\lambda+\beta}\right)$. Because it is a function, it then belongs to $\Delta_{1}\left(L_{\lambda+\beta}\right)$.

For $\omega \eta \leq \delta$, let $H_{\omega \eta}=H \uparrow\left(\boldsymbol{K}_{\omega \eta} \times\{\varnothing \mid \rho(\varnothing)=0\right.$ or $\left.|\varnothing|<\eta\}\right)$. For $\delta \leq \eta<$ $\lambda+\beta$ and $k<\omega$, let $H^{\omega \eta+k}=H \uparrow(\boldsymbol{K} \times\{\varnothing|| \varnothing \mid<\omega \eta+k$ or $\rho(\varnothing)=0\})$. We shall find $\Sigma_{1}$ formulas $\Phi_{1}(f)$ and $\Phi_{2}(f, K, \varnothing, i)$ such that for $\omega \xi \leq \delta$ :

(12) if $\eta<\xi, H_{\omega \eta} \in L_{\omega \xi}$;

(13) $L_{\omega \xi} \vDash \Phi_{1}(f)$ iff for some $\eta<\xi, f=H_{\omega \eta}$;

(14) if $\xi$ is a successor, $H_{\omega \xi}(K, \varnothing)=i$ iff

$$
L_{\omega \xi} \vDash(\exists f)\left(\Phi_{1}(f) \& \Phi_{2}(f, K, \varnothing, i)\right) ;
$$

(15) if $\xi$ is a limit, $H_{\omega \xi}(K, \varnothing)=i$ iff

$$
L_{\omega \xi} \vDash(\exists f)\left(\Phi_{1}(f) \& f(K, \varnothing)=i\right) .
$$

We shall find a $\Sigma_{1}$ formula $\Phi_{3}(f)$ and for each $k<\omega$ a formula $\chi_{k}(f, K, \varnothing, i)$ such that:

(16) for $k<\omega H^{\delta+k}(K, \varnothing)=i$ iff $L_{\delta} \vDash(\exists f)\left(\Phi_{1}(f) \& \chi_{k}(f, K, \varnothing, i)\right)$; and for $\xi$ such that $\delta<\xi \leq \lambda+\beta$;

(17) for $\delta \leq \eta<\xi$ and $k<\omega$, $H^{\omega \eta+k} \in L_{\xi}$;

(18) $L_{\xi} \models \Phi_{3}(f)$ iff for some $\eta$ and $k, \delta \leq \eta<\xi$ and $k<\omega, f=H^{\omega \eta+k}$;

(19) for $\xi$ such that $\delta<\xi<\lambda+\beta$ and $k<\omega$,

$$
H^{\omega \xi+k}(K, \varnothing)=i \quad \text { iff } L_{\xi} \vDash(\exists f)\left(\Phi_{3}(f) \& \chi_{k}(f, K, \varnothing, i)\right) ;
$$

and finally for $\varnothing \in \Pi_{0}$;

(20) $H(K, \varnothing)=i$ iff $L_{\lambda+\beta} \vDash(\exists f)\left(\Phi_{3}(f) \& \chi(f, K, \varnothing, i)\right)$.

As a first approximation to $\Phi_{1}(f)$, consider 
(21) $f$ is a function into $2 \&(\exists \eta)\left(\omega \eta\right.$ exists $\& \operatorname{dom}(f)=\boldsymbol{K}_{\omega \eta} \times\{\varnothing|| \varnothing \mid<\eta\} \&$ $(\forall\langle K, \varnothing\rangle \in \operatorname{dom} f)$ :

(1) $\rho(\varnothing)=0 \Rightarrow(f(K, \varnothing)=1$ iff $\varnothing$ is true),

(2) $(\forall k \in \omega)\left(\varnothing=\left\ulcorner\underline{B}_{0}(\underline{k})\right\urcorner \Rightarrow(f(K, \varnothing)=1\right.$ iff $\left.\ldots)\right)$,

(6) $(\forall \phi \in\{\varnothing|0<| \varnothing \mid<\eta\})\left(\varnothing=\left\ulcorner\neg \psi^{\prime} \Rightarrow\left(f(K, \varnothing)=1\right.\right.\right.$ iff $\left(\forall K^{\prime}\right)\left(K^{\prime}\right.$ extends $\left.\left.K \Rightarrow f\left(K^{\prime}, \psi\right)=0\right)\right)$ ),

(8) $(\forall \xi)(\forall \psi \in\{\varnothing|| \varnothing \mid<\eta\})\left(\varnothing=\left\ulcorner\left(\exists x^{\xi}\right) \psi^{\urcorner} \Rightarrow\left(f(K, \varnothing)=1\right.\right.\right.$ iff $\left(\exists c \in C_{\xi}\right)$ $\left.\left.\left(f\left(K, \phi\left(X^{\xi} / c\right)=1\right)\right)\right)\right)$ :).

What are the failings of (1)? The ' $(\forall \xi)$ ' in clause (8) is unrestricted. But for $|\varnothing|<$ $\eta$, if $\varnothing=\left(\exists^{\xi} x\right) \psi, \xi \leq \rho(\varnothing) \leq|\varnothing|<\eta$. Thus it may be replaced by ' $(\forall \xi<\eta)$ '. More seriously, the quantifier over conditions in clause (6) is not only unrestricted within $L_{\omega \xi}$ for $\omega \xi \leq \delta$, but if $\omega \xi<\delta$, its intended range includes more than $L_{\omega \xi}$. We shall show that in fact it may be replaced by ' $\left(\forall K^{\prime} \in \boldsymbol{K}_{\omega \eta}\right)$ '. This shall require several facts about modified Steel conditions due, essentially, to Steel [10].

If $x$ and $y$ are modified Steel conditions and $\eta$ is a limit ordinal, then $x$ is an $\eta$ retag of $y$ iff: $\operatorname{dom}(x)=\operatorname{dom}(y)$; if $x(\sigma)<\eta$ then $x(\sigma)=y(\sigma)$; if $x(\sigma) \geq \eta$ then $y(\sigma) \geq \eta$. Notice that 'is an $\eta$-retag of' is symmetric.

Retagging Lemma. Suppose that $x, x^{\prime}$ and $y$ are modified Steel conditions, $x^{\prime}$ extends $x$, and $\xi<\eta$ are two limit ordinals. If $y$ is an $\eta$-retag of $x$ then some modified Steel condition $y^{\prime}$ extends $y$, and is a $\xi$-retag of $x^{\prime}$.

Proof. Let

$$
r(\sigma)=\left\{\begin{array}{l}
0 \text { if } x^{\prime}(\sigma)<\xi \\
1+\max \left\{r\left(\sigma^{\wedge} j\right) \mid \sigma^{\wedge} j \in \operatorname{dom} x^{\prime}\right\} \text { otherwise. }
\end{array}\right.
$$

Clearly $\operatorname{dom}(r)=\operatorname{dom}\left(x^{\prime}\right)$, since $\operatorname{dom}\left(x^{\prime}\right)$ is finite and wellfounded under $\prec$, where $\sigma \prec \tau$ iff $\tau$ properly extends $\sigma$.

Let

$$
y^{\prime}(\sigma)=\left\{\begin{array}{l}
x^{\prime}(\sigma) \text { if } r(\sigma)=0, \\
1+\max \left\{y^{\prime}\left(\sigma^{\wedge} j\right) \mid \sigma^{\wedge} j \in \operatorname{dom} x^{\prime}\right\} \quad \text { if } r(\sigma) \neq 0 \text { and } \sigma \notin \operatorname{dom} y, \\
y(\sigma) \text { otherwise. }
\end{array}\right.
$$

Clearly $\operatorname{dom}\left(y^{\prime}\right)=\operatorname{dom}\left(x^{\prime}\right)$.

Claim. If $\sigma \prec \tau \in \operatorname{dom}\left(y^{\prime}\right)$ then $y^{\prime}(\sigma)>y^{\prime}(\tau)$.

This is straightforward unless $\sigma \in \operatorname{dom}(y)$ and $\tau \notin \operatorname{dom}(y)$. Then, by induction on $r(\tau), y^{\prime}(\tau) \leq x^{\prime}(\tau)$. But $x^{\prime}(\tau)<x^{\prime}(\sigma)=x(\sigma)$. If $x(\sigma)<\eta, x(\sigma)=y(\sigma)=y^{\prime}(\sigma)$, yielding $y^{\prime}(\tau)<y^{\prime}(\sigma)$. If $x(\sigma) \geq \eta, y^{\prime}(\sigma) \geq \eta$. But by induction on $r(\tau), y^{\prime}(\tau)<\xi+$ $\omega \leq \eta$. So again $y^{\prime}(\tau)<y^{\prime}(\sigma)$. Thus $y^{\prime}$ is a modified Steel condition. Clearly $y^{\prime}$ is a $\xi$-retag of $x^{\prime}$. Suppose $\sigma \in \operatorname{dom}(y)$. If $r(\sigma) \neq 0, y^{\prime}(\sigma)=y(\sigma)$. If $r(\sigma)=0, y^{\prime}(\sigma)=$ $x^{\prime}(\sigma)=x(\sigma)=y(\sigma)$ because $x(\sigma)<\xi<\eta$ and $y$ is an $\eta$-retag of $x$. Thus $y^{\prime}$ extends $y$. Note that if $\eta \leq \operatorname{ht} y$, ht $\left(y^{\prime}\right)=\operatorname{ht}(y)$; if ht $(y)<\eta$, ht $\left(y^{\prime}\right)<\xi+\omega$. 
COROLlaRy 1. If $x^{\prime}$ and $x$ are modified Steel conditions, $x^{\prime}$ extending $x$, and $\xi$ is a limit ordinal, then there is a modified Steel condition $y$ extending $x$ such that $y$ is $a$ $\xi$-retag of $x$ and $\mathrm{ht}(y)<\max (\mathrm{ht}(x)+\omega, \xi+\omega)$.

Proof. Because $x$ is a $(\xi+\omega)$-retag of itself, the desired $y$ exists by the retagging lemma and the concluding remark in its proof.

Let condition $\langle P, Q, x, y\rangle$ be a $\xi$-retag of $\left\langle P, Q, x^{\prime}, y^{\prime}\right\rangle$ iff $x$ and $y$ are $\xi$-retags of $x$ and $y^{\prime}$ respectively. The previous lemma and corollary remain true when modified Steel conditions are replaced by conditions.

CoROllaRy 2. For $\varnothing \in \Pi_{0}$ such that $|\varnothing| \leq \xi$ or $\rho(\varnothing)=0$ and $K^{\prime}$ an $\omega \cdot \xi$-retag of $K: K H \varnothing$ iff $K^{\prime} H \varnothing$.

Proof. If $\rho(\varnothing)=0$, this is trivial. We now induce on $|\varnothing|$. Clearly $K H \underline{B}_{0}(\underline{k})$ iff $K^{\prime} H \underline{B}_{0}(\underline{k})$.

Similarly for the other base clauses. All induction steps except the one for negation are trivial. Suppose $K H \neg \varnothing$. Let $K_{0}$ extend $K$ such that $K_{0} H \varnothing$. Since $|\neg \varnothing| \leq \xi$, $|\varnothing|<\xi$. The retagging lemma provides $K_{0}^{\prime}$ extending $K^{\prime}$ which is an $\omega \cdot|\varnothing|$-retag of $K$. By induction hypothesis, $K_{0} H \varnothing$ iff $K_{0}^{\prime} H \varnothing$. Thus $K^{\prime} H \neg \varnothing$. The converse follows symmetrically.

Corollary 3. For $K \in \boldsymbol{K}_{\omega \xi}$ and $\varnothing$ such that $|\varnothing|<\xi, K H \neg \varnothing$ iff for any $K^{\prime} \in$ $\boldsymbol{K}_{\omega \xi}$, if $K^{\prime}$ extends $K, K^{\prime} H \varnothing$.

Proof. $(\Rightarrow)$ is clear. $(\Leftarrow)$ Suppose $K^{*}$ extends $K, K^{*} H \varnothing$. By Corollary 1 there is a $K^{\prime}$ extending $K, K^{\prime}$ an $\omega \cdot|\varnothing|$-retag of $K^{*}$, and $\operatorname{ht}\left(K^{*}\right)<\max (\mathrm{ht}(K)+\omega$, $\omega \cdot|\varnothing|+\omega) \leq \omega \xi$. Thus $K^{*} H \varnothing$ by Corollary 2 and $K^{*} \in \boldsymbol{K}_{\omega \xi}$. So the quantifier restriction in $(*)(6)$ may be introduced.

We now construct $\Phi_{2}$. Let $t$ be a $\Sigma_{1}$ term such that for any $\eta, L_{\omega(\eta+1)} \vDash t=\eta$. As a first approximation let $\Phi_{2}(f, K, \varnothing, i)$ be:

$(|\varnothing|<t \& f(K, \varnothing)=i) \vee\left(|\varnothing|=t \& \operatorname{dom}(f)=\left(\boldsymbol{K}_{\omega \cdot t} \times\{\varnothing|| \varnothing \mid<t\} \&\right.\right.$

(1) $\rho(\varnothing)=0 \Rightarrow(i=1$ iff $\varnothing$ is true $)$

(2) $(\forall k \in \omega)\left(\varnothing=\left\ulcorner\underline{B}_{0}(\underline{k})\right\urcorner \Rightarrow(i=1\right.$ iff $\left.\ldots)\right)$

(6) $(\forall \psi \in\{\varnothing|| \varnothing \mid<t\})\left(\varnothing=\ulcorner\neg \psi\urcorner \Rightarrow\left(i=1\right.\right.$ iff $\left.\left.\left(\forall K^{\prime} \in \boldsymbol{K}_{\omega t}\right)\left(f\left(K^{\prime}, \psi\right)=0\right)\right)\right)$ $:)$.

The arguments used in revising $(*)$ show that for $|\varnothing|=\eta$,

$$
L_{\omega(\eta+1)} \vDash \Phi_{1}(f) \& \Phi_{2}(f, K, \varnothing, i) \quad \text { iff } f=H_{\omega \eta} \text { and } H_{\omega(\eta+1)}(K, \varnothing)=i \text {. }
$$

We now prove (12)-(15) by simultaneous induction. (12) is vacuously true for $\xi=0$. For any $\xi$ such that $\omega \xi \leq \delta$, if (12) is true, so is (13). Then so are (14) and (15). Thus $H_{\omega \xi} \in L_{\omega \xi+1}$, implying (12) for $\xi+1$. If $\xi$ is a limit, (12) holds for $\xi$ induction. Thus (12)-(15) are all true. Consequently $H_{\delta} \in \Sigma_{1}\left(L_{\delta}\right)$ and thus $H_{\delta} \in L_{\delta+1}$. If $\delta=\lambda+\beta$, this proves Sublemma 2 .

If $\delta<\lambda+\beta$, notice that $K \in L_{\delta+1} \subseteq L_{\lambda+\beta} . \Phi_{3}(f)$ may be taken to have the form:

$$
\left(f=H_{\delta}\right) \vee(\exists \eta)(\exists k<\omega)\left(\delta \leq \eta \& \operatorname{dom}(f)=K \times\{\varnothing|| \varnothing \mid<\omega \eta+k\} \& \Phi^{\prime}(f)\right),
$$

where the construction of $\Phi^{\prime}$ is easy. Notice that the quantifier $(\forall K)$ in clause (6) 
may be restricted to $K$. Let $\chi_{0}(f, K, \varnothing, i)$ be $f(k, \varnothing)=i$. Suppose $\chi_{k}$ has been constructed. Let $\chi_{k+1}(f, K, \varnothing, i)$ be

(5) $(\forall \phi)\left(\varnothing=\left\ulcorner-\psi^{\prime} \Rightarrow\left(i=1\right.\right.\right.$ iff $\left(\forall K^{\prime}\right.$ extending $\left.\left.\left.K\right)\left(X_{k}\left(F, K^{\prime}, \phi, 0\right)\right)\right)\right) \&$

(6) $(\forall \psi)\left(\forall \psi^{\prime}\right)\left(\varnothing=\left\ulcorner\psi \wedge \phi^{\prime}\right\urcorner \Rightarrow\left(i=1\right.\right.$ iff $\left.\left(X_{k}(F, K, \psi, 1) \&\left(X_{k}\left(F, K, \psi^{\prime}, 1\right)\right)\right)\right)$, :

(16) is clear, using (13), yielding (17) for $\xi=\delta+1$. Assume (17) for arbitrary $\xi$. By construction, (18) and (19) are true for $\xi$. Thus (17) is true for $\xi+1$. If $\xi$ is a limit and (17) is true for all $\xi^{\prime}<\xi$, (17) is true for $\xi$. Thus (17)-(19) are true for all $\xi$ such that $\delta<\xi \leq \lambda+\beta$. So (20) is also true, proving that $H \uparrow\left(\boldsymbol{K} \times \Pi_{0}\right) \in$ $\Sigma_{1}\left(L_{\lambda+\beta}\right)$. Sublemma 2 is proven.

Suppose $\left\langle K_{i}\right\rangle_{i \in \omega}$ is a $\Sigma_{n}$ generic sequence, i.e. for each $i \in \omega, K_{i+1}$ extends $K_{i}$ and for every $\varnothing \in \Sigma_{n}$ there is an $i$ such that $K_{i}$ decides $\varnothing$. Where $K_{i}=\left\langle P_{i}, Q_{i}\right.$, $\left.x_{i}, y_{i}\right\rangle$, let $T_{0}=\bigcup x_{i}, T_{1}=\bigcup y_{i}, B_{1}=\operatorname{dom}\left(T_{0}\right)$ and $C_{1}=\operatorname{dom}\left(T_{1}\right)$. By the usual forcing = truth lemma, for any $k \in \omega, k \in B_{1}$ iff for some $i, K_{i}+\underline{B}_{1}(\underline{k})$, and similarly for $C_{1}$. By definition of modified Steel conditions, $T_{0}: \subseteq$ seq $-\{\langle\rangle\} \rightarrow \delta$ such that if $\sigma \prec \tau \in \operatorname{dom}\left(T_{0}\right), \sigma \in \operatorname{dom}\left(T_{0}\right)$ and $T_{0}(\sigma)>T_{0}(\tau)$. Similarly for $T_{1}$. Thus $\left\langle B_{1}, \prec\right\rangle$ and $\left\langle C_{1},\langle\rangle\right.$ are wellfounded.

Sublemma 3. If $\sigma \in B_{1}$, for any $\xi,|\sigma|_{B_{1}}=\xi$ iff for some $i, x_{i}(\sigma)=\xi$.

Furthermore, the order type of $\left\langle B_{1}, \prec\right\rangle=\delta$. Similarly for $C_{1}$ and $y_{i}$. Proof by induction on $|\sigma|_{B_{1}}$. Suppose $|\sigma|_{B_{1}}=\xi$. For some $i, \sigma \in \operatorname{dom}\left(x_{i}\right)$ and $K_{i} H$ " $|\underline{\sigma}|_{B_{1}}=\xi "$. By the induction hypothesis $(\Leftarrow),|\sigma|_{B_{1}} \leq x_{i}(\sigma)$. So $x_{i}(\sigma) \geq \xi$. If $x(\sigma)>\xi$, let $K=$ $\left\langle P_{i}, Q_{i}, x_{i} \cup\left\{\left\langle\sigma^{\wedge} j, \xi\right\rangle\right\}, y_{i}\right\rangle$, for some $j$ such that $\sigma^{\wedge} j \notin \operatorname{dom}\left(x_{i}\right)$, and form another generic sequence extending $K$ and yielding $\hat{B}_{1}$ in place of $B_{1}$. By choice of $i$ and forcing $=$ truth, $|\sigma|_{\hat{B}_{1}}=\xi$. But by the induction hypothesis $(\Leftarrow),\left|\sigma^{\wedge} j\right|_{\hat{B}_{1}}=\xi$. Contradiction. Thus $x_{i}(\sigma)=\xi$. Now suppose that $x_{i}(\sigma)=\xi$. If $|\sigma|_{\hat{B}_{1}}>\xi$, for some $j, \sigma^{\wedge} j \in B_{1}$ and $\left|\sigma^{\wedge} j\right|_{\hat{B}_{1}} \geq \xi$. By the induction hypothesis $(\Rightarrow)$ there is an $i^{\prime} \geq i$ such that $x_{i^{\prime}}\left(\sigma^{\wedge} j\right) \geq \xi$, which is impossible. If $\eta=|\sigma|_{B_{1}}<\xi$, select $i^{\prime} \geq i$ such that $K_{i}, H$ " $|\underline{\sigma}|_{B_{1}}=\eta$ ". Where $K=\left\langle P_{i^{\prime}}, Q_{i^{\prime}}, x_{i} \cup\left\{\left\langle\sigma^{\wedge} j, \eta\right\rangle\right\}, y_{i^{\prime}}\right\rangle$ for $j$ such that $\sigma^{\wedge} j \notin \operatorname{dom} x_{i}$, form another generic sequence extending $K$ and yielding $\hat{B}_{1}$ in place of $B$. By choice of $i$ and forcing $=$ truth, $|\sigma|_{\hat{B}_{1}}=\eta$. By the induction hypothesis $(\Leftarrow),\left|\sigma^{\wedge} j\right|_{\hat{B}_{1}}=\eta$.Contradiction. Thus $|\sigma|_{B_{1}}=\xi$. Suppose $\sup \left\{|\sigma|_{B_{1}} \mid \sigma \in B_{1}\right\}=\xi<\delta$. For some $i, K_{i} H$ "sup $\left\{|\sigma|_{B_{1}} \mid \underline{B}_{1}(\sigma)\right\}=\xi$ ". Let $K=\left\langle P_{i}, Q_{i}, x_{i} \cup\{\langle\langle j\rangle, \xi+1\rangle\}\right.$, $\left.y_{i}\right\rangle$ for $\langle j\rangle \in \operatorname{dom}\left(x_{i}\right)$. Form a generic sequence extending $K$ and yielding $\hat{B}_{1}$ in place of $B_{1}$. By forcing = truth, $\sup \left\{|\sigma|_{\hat{B}_{1}} \mid \sigma \in \hat{B}_{1}\right\}=\xi$. But by previous parts of this sublemma, $|\langle j\rangle|_{\hat{B}_{1}}=\xi+1$. Contradiction. A symmetric argument applies to $C_{1}$.

We now construct $B$ and $C$. Let $\left\langle A_{i}\right\rangle_{i \in \omega}$ and $\left\langle\varnothing_{i}\right\rangle_{i \in \omega}$ be enumerations of $L_{\lambda} \cap 2^{\omega}$ and the $\Sigma_{n}$ sentences of $\boldsymbol{L}$, both members of $\Delta_{n+1}\left(L_{\lambda+\beta}\right)$. Such enumerations exist by the corollary to the Fundamental Theorem. Define $\left\langle K_{i}\right\rangle_{i \in \omega} \in \Delta_{n+1}\left(L_{\lambda+\beta}\right)$ by:

$$
\begin{aligned}
K_{0} & =\langle\mathrm{id}, \mathrm{id}, \wedge, \wedge\rangle ; \\
K_{2 i+1} & =\text { the }\left\langle_{L} \text {-least condition extending } K_{2 i} \text { and deciding } \varnothing_{i} ;\right. \\
K_{2 i+2} & =\left\langle P * A_{i}, Q * A_{i}, x, y\right\rangle \quad \text { where } K_{2 i+1}=\langle P, Q, x, y\rangle .
\end{aligned}
$$


Letting $K_{i}=\left\langle P_{i}, Q_{i}, x_{i}, y_{i}\right\rangle$, let $B_{0}=\bigcap_{i<\omega}\left[P_{i}\right], C_{0}=\bigcap_{i<\omega}\left[Q_{i}\right]$ and $B_{1}, C_{1}, T_{0}$ and $T_{1}$ as above. Let $B=B_{0} \oplus B_{1}$ and $C=C_{0} \oplus C_{1}$. By the standard argument, if $f \leq_{T} B$ and $f \leq_{\top} C, f \in L_{\lambda+\beta}$, and so $f \in L_{\lambda}$. By the even stages of the construction, for any $f \in L_{\lambda}, f \leq_{T} B_{0}$ and $f \leq_{T} C_{0}$. Thus (1) is true. Because $\delta<\omega_{1}^{B_{1}}$ and $\delta<\omega_{1}^{C_{1}}$, (9) is true. Because $\left\langle K_{i}\right\rangle_{i \in \omega} \in \Delta_{n+1}\left(L_{\lambda+\beta}\right)$, there is an $M$, a term-model copy of $L_{\beta}[B, \oplus C]$, such that the $\operatorname{Th}_{n}(M) \in \Delta_{n+1}\left(L_{\lambda+\beta}\right)$. Because $\Delta_{n+1}\left(L_{\lambda+\beta}\right)$ contains a counting of the set of terms $C$, the preimage of $\varepsilon_{M}$ under this counting is the desired $E_{\beta}[B \oplus C]$, verifying (8).

We now prove Lemma 2 for Case 3. Recall that $f \in \omega^{\omega}-L_{\lambda}$. The language $L$ is as before. We now extend Sublemma 1 to this setting.

Sublemma 4. $\varnothing\left(x^{0}, y^{0}\right) \in \Sigma_{n}, K \in \boldsymbol{K}$. There are $k<\omega$ and $K^{\prime}$ extending $K$ such that either $K^{\prime} H \neg\left(\exists y^{0}\right) \varnothing\left(\underline{k}, y^{0}\right)$ or for some $m \neq f(k), K^{\prime} \vDash \varnothing(\underline{k}, \underline{m})$.

Proof. Suppose not. Then for any $k \in \omega$ and any $K^{\prime}$ extending $K$ there is a $K^{*}$ extending $K^{\prime}$ s.t. $K^{*}+\left(\exists y^{0}\right) \varnothing\left(\underline{k}, y^{0}\right)$, and for any such $K^{\prime}$, if $K^{\prime} H \varnothing(\underline{k}, \underline{m})$ then $f(k)=m$. This gives us a $\Sigma_{n}$ definition of $f$ over $L_{\lambda+\beta}$. Thus $f \in \Delta_{n}\left(L_{\lambda+\beta}\right)$. But $L_{\lambda+\beta}=\Delta_{n}$ CA. So $f \in L_{\lambda+\beta}$. Contradiction. Select such a $k$ and $K^{\prime}$ and call them $k(K, \varnothing)$ and $K^{\prime}(K, \varnothing)$ respectively.

We may now construct $B$ and $C$. Let $\left\langle A_{i}\right\rangle_{i \in \omega}$ and $\left\langle\varnothing_{i}\right\rangle_{i \in \omega}$ enumerate $L_{\lambda} \cap 2^{\omega}$ and the $\Sigma_{n}$ sentences of $L$. Let $\left\langle K_{i}\right\rangle_{i \in \omega}$ be such that:

$K_{0}=\langle$ id, id, $\wedge, \wedge\rangle$;

$K_{3 i+1}$ decides $\varnothing_{i}$;

$K_{3 i+2}\left(P * A_{i}, Q * A_{i}, x, y\right)$ where the $K_{3 i+1}=(P, Q, x, y)$;

$K_{3 i+3}=K_{3 i+2}$ if $\varnothing_{i}$ does not contain exactly the free variables $x^{0}$ and $y^{0}$, $K^{\prime}\left(K_{3 i+2}, \varnothing_{i}\right)$ otherwise.

Form $B_{0}, B_{1}, C_{0}, C_{1}, B, C$ from $\left\langle K_{i}\right\rangle_{i \in \omega}$ as before. As with Lemma 1, (1) and (9) are true. Suppose $f \in \Sigma_{n}\left(L_{\beta}[B \oplus C]\right)$. Then $f$ is defined over $L_{\beta}[B \oplus C]$ by some $\varnothing_{i}\left(x^{0}, y^{0}\right)$. For some $j, K_{j} H \varnothing_{i}(\underline{k}, \underline{f}(\underline{k}))$ where $k=k\left(K_{3 i+2}, \varnothing_{i}\right)$. But either $K_{i} H \neg\left(\exists y^{0}\right) \varnothing_{i}\left(\underline{k}, y^{0}\right)$ or $K_{i} H \varnothing_{i}(\underline{k}, \underline{m})$ for $m \neq f(k)$. Contradiction. Thus (10) is also true.

Again we note that if $\underline{d} \leq \underline{0}^{(\lambda)},(\underline{b}, \underline{c})$ could be constructed so that $(\underline{b} \vee \underline{c})^{(G(\lambda))} \leq$ $\underline{0}^{(\lambda)}$, thus $(\underline{b} \vee \underline{c})^{(G(\lambda))}=\underline{0}^{(\lambda)}$ by Lemma 3 .

We now turn to Lemma 3. Suppose that $(\underline{b}, c)$ is $I_{\lambda}$-exact, $B \in \underline{b}$ and $C \in \underline{c}$. Let $J(\lambda)=\omega \gamma$. In Cases 1 and 2 we want to construct a real $\operatorname{Th}_{F(J(\lambda))}\left(E_{\gamma}\right)$ which is recursive in $(B \oplus C)^{(G(\lambda))}$. By an easy modification of Definition 8 from [1], there is an operator $*$ on $2^{\omega} \times \omega$ such that (i) $(X, y)^{*} \leq_{\mathrm{T}} X^{(\omega)}$ uniformly in $y$, and (ii) for any $E_{\xi}$ and $b \in \omega$ such that for no $x \in \operatorname{Fld}\left(E_{\xi}\right),(x)_{1}=b,\left(\operatorname{Th}_{0}\left(E_{\xi}\right), b\right)^{*}=$ $\mathrm{Th}_{0}\left(E_{\xi+1}\right)$ for an $E_{\xi+1}$ extending $E_{\xi}$. We also note that the relation $X=Y^{(\omega)}$ is $\Pi_{1}^{0}$ over $2^{\omega} \times 2^{\omega}$ (although it is only $\Pi_{2}^{0}$ over $P \omega \times P \omega !$ ).

Case 1.1. $F(\lambda)=0$ and $G(\lambda)=2$. If $\gamma=\gamma^{\prime}+1, \gamma^{\prime}$ is an $L$-index for otherwise $\lambda$ falls under Case 3. Applying the corollary to the Fundamental Theorem to $\gamma^{\prime}$, there is a real $\operatorname{Th}_{0}\left(E_{\gamma^{\prime}}\right) \in L_{\gamma}$. $E_{\gamma^{\prime}}$ may be chosen so that for any $x \in \operatorname{Fld}\left(E_{\gamma^{\prime}}\right),(x)_{1} \neq$ 0 . Let $\mathrm{Th}_{0}\left(E_{\gamma}\right)=\left(\mathrm{Th}_{0}\left(E_{\gamma^{\prime}}\right), 0\right)^{*} \leq_{\mathrm{T}} \mathrm{Th}_{0}\left(E_{\gamma^{\prime}}\right)^{(\omega)}$. By results in [4], $(B \oplus C)^{(2)}$ can compute a nice parametrization of $L_{r} \cap \omega^{\omega}$. Thus $\operatorname{Th}_{0}\left(E_{\gamma^{\prime}}\right)^{(\omega)} \leq_{\top}(B \oplus C)^{(2)}$.

Now suppose that $\gamma$ is a limit. Select a $\gamma^{\prime}<\gamma$ such that $\left[\gamma^{\prime}, \gamma\right)$ contains no $M$-gaps. As before, select a real $\mathrm{Th}_{0}\left(E_{\gamma^{\prime}}\right) \in L_{\gamma^{\prime}+1}$. By choice of $\gamma^{\prime}$, there is a linear 
system of notation $R \in L_{\gamma^{\prime}+1}$ of height $\left(\gamma-\gamma^{\prime}\right)$. Working over $L_{\gamma}$ we construct a sequence $\left\langle\operatorname{Th}_{0}\left(E_{x}\right)\right\rangle_{x \in \operatorname{Fld}(R)}$, each $E_{x}$ is an $E_{|x|_{R}}$, as follows.

$$
\begin{aligned}
& \operatorname{Th}_{0}\left(E_{x}\right)=\left\{\begin{array}{l}
\operatorname{Th}_{0}\left(E_{\gamma^{\prime}}\right) \quad \text { if }|x|_{R}=0 ; \\
\left(\operatorname{Th}_{0}\left(E_{y}\right), y\right)^{*} \quad \text { if }|x|_{R}=|y|_{R}+1 ; \\
\bigcup_{y<R} \operatorname{Th}_{0}\left(E_{y}\right) \quad \text { if }|x|_{R} \text { is a limit. }
\end{array}\right. \\
& \operatorname{Th}_{0}\left(E_{\gamma}\right)=\bigcup_{x \in \operatorname{Fld}(R)} \operatorname{Th}_{0}\left(E_{x}\right) .
\end{aligned}
$$

To show that $\mathrm{Th}_{0}\left(E_{\gamma}\right) \leq_{\mathrm{T}}(B \oplus C)^{(2)}$, we introduce another such sequence.

$$
\begin{aligned}
& H_{x}(R)= \begin{cases}\mathrm{Th}_{0}\left(E_{\gamma^{\prime}}\right) & \text { if }|x|_{R}=0, \\
H_{y}(R)^{(\omega)} & \text { if }|x|_{R}=|y|_{R}+1, \\
\left\{\langle y, z\rangle \mid z \in H_{y}(R) \& y<_{R} x\right\} .\end{cases} \\
& H(R)=\left\{\langle x, z\rangle \mid z \in H_{x}(R) \& x \in \operatorname{Fld}(R)\right\} .
\end{aligned}
$$

By induction along $R, \mathrm{Th}_{0}\left(E_{x}\right) \leq_{\mathrm{T}} H_{x}(R)$ uniformly in $x$ and $H_{x}(R) \in L_{\gamma}$ for each $x \in \operatorname{Fld}(R)$. Thus $\operatorname{Th}_{0}\left(E_{\gamma}\right) \leq_{\mathrm{T}} H(R)$ and $H(R) \in \Delta_{1}^{1}\left(L_{\gamma} \cap \omega^{\omega}\right)$. Again because $(B \oplus C)^{(2)}$ computes a nice parametrization of $L_{\tau} \cap \omega^{\omega}, H(R) \leq_{\top}(B \oplus C)^{(2)}$.

Case 1.2. $\lambda=\gamma, F(\lambda)=1$ and $G(\lambda)=3$. We use the previous argument with a twist. Let $\gamma^{\prime}<\gamma$ be maximum such that $\gamma^{\prime}$ is admissible or a limit of admissibles. $L_{\lambda}$ contains no system of notation for $\lambda$. But because $(B \oplus C)^{(2)}$ computes a nice parametrization of $L_{\lambda} \cap \omega^{\omega}$, there is a linear system of notation $R$ of $\lambda=\lambda-\gamma^{\prime}$ such that $H(R) \leq_{\top}(B \oplus C)^{(2)}$ and each initial segment of $R$ belongs to $L_{\lambda}$. This follows from Theorem 2 of [4], replacing the ordinary jump by the $\omega$-jump. Select $\operatorname{Th}_{0}\left(E_{\gamma^{\prime}}\right) \in L_{\gamma^{\prime}+1}$ and construct $\left\langle\operatorname{Th}_{0}\left(E_{x}\right)\right\rangle_{x \in \mathrm{Fld}(R)}$ and $\mathrm{Th}_{0}\left(E_{\lambda}\right)$ as before, with $E_{x}$ an $E_{\gamma^{\prime}+|x|_{R}}$. Again $\mathrm{Th}_{0}\left(E_{\lambda}\right) \leq_{\mathrm{T}} H(R) \leq_{\mathrm{T}}(B \oplus C)^{(2)}$. But then $\mathrm{Th}_{1}\left(E_{\lambda}\right) \leq_{\mathrm{T}} \mathrm{Th}_{0}\left(E_{\lambda}\right)^{\prime} \leq_{\mathrm{T}}$ $(B \oplus C)^{(3)}$.

Case 2. $\lambda=\gamma$. Let $F(\lambda)=n, G(\lambda)=n+3$. Again, by a slight revision of Theorem 3 of [4], we may select a linear system of notation $R$ for $\lambda$, such that $H(R)$ $\leq_{\top}(B \oplus C)^{(3)}$. Select $\mathrm{Th}_{0}\left(E_{0}\right) \in L_{1}$. We construct $\left\langle\mathrm{Th}_{0}\left(E_{x}\right)\right\rangle$, with $E_{x}$ an $E_{|x|_{R}}$, and $\mathrm{Th}_{0}\left(E_{\lambda}\right)$ as before. Again $\mathrm{Th}_{0}\left(E_{\lambda}\right) \leq_{\mathrm{T}} H(R)$. Thus $\mathrm{Th}_{n}\left(E_{\lambda}\right) \leq_{\mathrm{T}} \mathrm{Th}_{0}\left(E_{\lambda}\right)^{(n)} \leq_{\mathrm{T}}$ $(B \oplus C)^{(G(\lambda))}$.

Case 3. $\lambda=\gamma, F(\lambda)=G(\lambda)=\omega \beta+n$. The argument divides into two subcases. Suppose $\beta<\lambda+\beta$. Thus $\beta<\omega \lambda$, $\operatorname{Ind}^{(\underline{b} \vee c)}(G(\lambda))=G(\lambda)$. We wish to find an $E_{\lambda+\beta}$ such that $\operatorname{Th}_{n}\left(E_{\lambda+\beta}\right) \in \Delta_{n+1}\left(L_{\beta}[B \oplus C]\right)$. By the argument for Case 2, there is a real $\mathrm{Th}_{0}\left(E_{\lambda}\right) \leq_{\mathrm{T}}(B \oplus C)^{(3)}$, and so belonging to $L_{1}[B \oplus C]$. Let $R$ be a linear system of notation for $\beta$ such that $R \leq_{T}(B \oplus C)^{(3)}$ and all initial segments of $R$ belong to $L_{\lambda}$. Within $L_{\beta}[B \oplus C]$ we construct a sequence $\left\langle\operatorname{Th}_{0}\left(E_{x}\right)\right\rangle_{x \in \operatorname{Fld}(R)}$ starting with $\mathrm{Th}_{0}\left(E_{x}\right)$ and such that $E_{x}$ is an $E_{\lambda+|x|_{R}}$. $\operatorname{Th}_{0}\left(E_{\lambda+\beta}\right)=\bigcup_{x \in \operatorname{Fld}(R)} \operatorname{Th}_{0}\left(E_{x}\right)$ as before; so $\mathrm{Th}_{0}\left(E_{\lambda+\beta}\right) \leq_{\mathrm{T}} H(R) . H(R) \in \Delta_{1}\left(L_{\beta}[B \oplus C]\right)$; so $H(R)^{(n)} \in \Delta_{n+1}\left(L_{\beta}[B \oplus c]\right)$ and $\mathrm{Th}_{n}\left(E_{\lambda+\beta}\right) \leq_{\mathrm{T}} \mathrm{Th}_{0}\left(E_{\lambda+\beta}\right)^{(n)} \leq_{\mathrm{T}} H(R)^{(n)}$.

Now suppose that $\beta=\lambda+\beta$. Let $\operatorname{Ind}^{(\underline{b} \vee c)}(G(\lambda)) \omega \cdot \delta+m$. The strategy used up to now is no longer available, for we cannot count on there being a system of 
notation for $\beta$ belonging to $L_{1}[B \oplus C]$. Let $\hat{L}=\left\{\langle\xi, x\rangle \mid x \in L_{\xi+1}-L_{\xi}\right.$ for $\xi<$ $\lambda+\beta\}$ and let $\langle\xi, x\rangle \hat{\epsilon}\langle\eta, y\rangle$ iff $x \in y$. Thus $\langle\hat{L}, \hat{\epsilon} \uparrow(\hat{L} \times \hat{L})\rangle$ is isomorphic to $\left\langle L_{\lambda+\beta}, \epsilon\right\rangle$ and $\hat{L} \in \Delta_{1}\left(L_{\beta}[B \oplus C]\right)$. Select $\operatorname{Th}_{m}\left(E_{\delta}[B \oplus C]\right) \in(\underline{b} \vee \underline{c})^{(G(\lambda))}$. Let $E_{\lambda+\beta}$ be the copy within $E_{\delta}[B \oplus C]$ of $\langle\hat{L}, \hat{\epsilon} \uparrow(\hat{L} \times \hat{L})\rangle$. Because $\omega \beta+n \leq \omega \delta+m$, $\mathrm{Th}_{n}\left(E_{\lambda+\beta}\right) \leq_{\mathrm{T}} \mathrm{Th}_{m}\left(E_{\delta}[B \oplus C]\right)$.

§3. Defining $\lambda \xi . \underline{0}^{(\xi)}$ inductively. $\lambda \xi . \underline{0}^{(\xi)}$ has been defined set-theoretically. In [6] $\left(\lambda \xi . \underline{0}^{(\xi)}\right) \uparrow \beta_{0}$ is shown to have a sort of degree-theoretic inductive definition over $\left\langle\mathscr{D} ; \leq,{ }^{\prime}\right\rangle$; viz. there is a sequence of formulae $\left\langle\psi_{i}(x)\right\rangle_{i<\omega}$ in the language of $\left\langle\mathscr{D} ; \leq\right.$, , $\left.^{\prime} I\right\rangle$ such that for any $\lambda<\beta_{0}$ there is an $i$ such that $\left\langle\mathscr{D} ; \leq,{ }^{\prime}, I_{\lambda}\right\rangle \vDash$ $(\exists x) \psi_{i}(x)$ and for the least such $i,\left\langle\mathscr{D} ; \leq,{ }^{\prime}, I_{\lambda}\right\rangle \vDash \underline{0}^{(\lambda)}=(\supset x) \phi_{i}(x)$. Allowing the appearance of $\lambda \underline{a} . \underline{a}^{(\xi)}$ for $\xi<\beta_{0}$ in the structure, we can bootstrap up to a larger initial segment of $\lambda \xi . \underline{0}^{(\xi)}$. How far may this be iterated? We define a sequence of suchinitial segments $\left\langle d_{i}\right\rangle_{i \leq \omega}$ as follows.

Given any partial function $d$ on ordinals, let $\operatorname{dom}^{*}(d)$ be the maximal initial segment of the ordinals on which $d$ is defined.

$d_{0}(n)=\underline{0}^{(n)}$ for $n \in \omega$.

$d_{i+1}(\lambda)=$ the least member of $\left\{d_{i}^{a}\left(\mu_{\lambda}\right) \mid \underline{a}\right.$ is $I_{\lambda}$-exact $\}$ if for every $I_{\lambda}$-exact $\underline{a}, \mu_{\lambda} \in$ $\operatorname{dom}^{*}\left(d_{i}^{a}\right)$; undefined, otherwise;

$d_{i+1}(\lambda+n)=d_{i+1}(\lambda)^{(n)}$.

$d_{\omega}(\lambda)=$ the least member of $\left\{d_{i}^{a}\left(\mu_{\lambda}\right) \mid \underline{a}\right.$ is $I_{\lambda}$-exact, $\left.i \in \omega\right\}$ if there is an $i \in \omega$ such that for every $I_{\lambda}$-exact $\underline{a}, \mu_{\lambda} \in \operatorname{dom}\left(d_{i}^{(a)}\right)$;

$d_{\omega}(\lambda+n)=d_{\omega}(\lambda)^{(n)}$.

Notice that $d_{i+1}(\lambda)=\max \left\{\underline{d} \mid\right.$ for any $I_{\lambda}$-exact $\left.\underline{a}, \underline{d} \leq d_{\underline{i}}^{a}\left(\mu_{\lambda}\right)\right\}$, under the above conditions for definition. So the definition of $d_{1}$ in terms of $d_{0}$ coincides with the inductive degree-theoretic definition of $\lambda \xi<\beta_{0} .0^{(\xi)}$ provided in [6].

$\alpha$ is a local $\kappa_{\xi}$ iff $L_{\alpha+1} \vDash \alpha=\aleph_{\xi}$. Let $\delta_{\xi}$ be the least local $\kappa_{\xi}$. Let $\delta_{<\omega}$ be the least $\alpha$ such that $L_{\alpha} \vDash(\forall n \in \omega)$ ( $\boldsymbol{\kappa}_{n}$ exists). Note that $\sup \left\{\delta_{n} \mid n \in \omega\right\}<\delta_{<\omega}<$ $\delta_{\omega}$. For $n \geq 1$, let $\lambda_{n}$ be that $\lambda$ such that $L_{\delta_{n}+1} \models \lambda=\kappa_{1}$. Let $\lambda_{\omega}$ be that $\lambda$ such that $L_{\delta<\omega} \vDash \lambda=\kappa_{1}$. Again, $\lambda_{\omega}<\lambda$ for that $\lambda$ such that $L_{\delta_{\omega}} \vDash \lambda=\kappa_{1}$.

TheOREM 4. For $\xi \leq \omega$, dom* $\left(d_{\xi}\right)=\lambda_{\xi}$.

This approach to defining $\underline{0}^{(\lambda)}$ in terms of $I_{\lambda}$ stops at $\lambda_{\omega}$. This follows from the following.

THEOREM 5. There is an $I_{\lambda_{\omega}}$-exact $\underline{\text { a such that } d_{\bar{\omega}}}\left(\mu_{\lambda_{\omega}}\right)$ is undefined. Thus if $d_{\omega+1}$ is defined in terms of $d_{\omega}$ just as $d_{i+1}$ is defined in terms of $d_{i}$, then $\operatorname{dom}^{*}\left(d_{\omega+1}\right)=\lambda_{\omega}$.

We simultaneously prove Theorem 4 and the following lemma.

LEMMA 4. Suppose that $[\lambda, \lambda+\alpha)$ is a maximal L-gap. $[\lambda, \lambda+\alpha)$ contains a local $\aleph_{i+1}$ iff for the some $I_{\lambda}$-exact $\underline{a}, \mu_{\lambda} \notin \operatorname{dom}^{*}\left(d_{i}^{a}\right)$.

Proof. $\lambda_{0}=\omega$. Thus for $\xi=0$, Theorem 4 is trivial. For $i=0$, by results from $\S 2$, both sides of the biconditional in Lemma 4 are true. $\lambda_{1}=\beta_{0}$. By results in $\S 2$, for $\xi=1$, Theorem 4 follows. Suppose $1<\xi<\omega$ and $\xi=i+1$. We assume as our induction hypothesis that for any $\underline{a}, \operatorname{dom}^{*}\left(d_{i}^{a}\right)=\lambda_{i}^{a}$. This is legitimate because this proof, though presented relative to $\underline{0}$, may be relativized to any degree. In what follows, we write $L_{\bar{\alpha}}^{\underline{a}}$ as $\left(L^{\underline{a}}, \alpha\right)$.

We first prove Lemma 4 for $i$ as above. $(\Leftarrow)$. Let $\underline{a}$ be $I_{\lambda}$-exact, $\mu_{\lambda} \notin \operatorname{dom} *\left(d_{i}^{a}\right)$. By induction hypothesis relativized to $\underline{a}$, $\operatorname{dom}^{*}\left(d_{i}^{a}\right)=\lambda_{i}^{a}$. So $\mu_{\lambda} \geq \lambda_{i}^{a}$. 
Claim. $\delta_{i}^{\frac{a}{i}} \in[\lambda, \lambda+\alpha)$.

First we show that $\lambda_{i}^{q} \leq \lambda+\alpha$. If $\lambda+\alpha<\lambda_{i}^{a}$, then $\alpha<\lambda_{i}^{\frac{a}{i}}$; so $\omega \alpha<\omega \lambda_{i}^{\frac{a}{i}}=$ $\lambda_{i}^{a}$. For some $n, \in \omega, \mu_{\lambda}=\omega \alpha+n$ and $\lambda_{i}^{a}$ is a limit; so $\mu_{\lambda}<\lambda_{i}^{a}$. Now we show that $\delta_{i}^{\underline{a}}<\lambda+\alpha$. Suppose $\lambda+\alpha \leq \delta_{i}^{\underline{a}}$. $\lambda+\alpha$ is an $L$-index. But $\left(L^{\underline{a}}, \delta_{i}^{\underline{a}}\right) \vDash \mathrm{ZF}^{-}$; thus $\left(L, \delta_{i}^{a}\right) \models \mathrm{ZF}^{-}$. So $\delta_{i}^{a}$ is not an $L$-index. Thus $\lambda_{i}^{\frac{a}{i}}<\lambda+\beta<\delta_{i}^{a}$. $L_{\lambda+\alpha+1}$ contains a wellordering of height $\lambda+\alpha$. So does $\left(L^{\underline{a}}, \lambda+\alpha+1\right)$. But $\left[\lambda_{i}^{a}, \delta^{\frac{a}{i}}+1\right)$ is an $L^{\underline{a}}$-gap. Contradiction. Therefore $\delta_{i}^{\underline{a}}<\lambda+\alpha$.

Claim. $\left[\lambda, \delta_{i}^{a}+1\right)$ contains a local $\aleph_{i+1}$. Let $\alpha_{1}, \ldots, \alpha_{i-1}$, be such that:

$$
\left(L^{\underline{a}}, \delta_{i}^{\underline{a}}+1\right) \models \alpha_{1}=\aleph_{1} \& \cdots \& \alpha_{i-1}=\aleph_{i-1} \& \delta_{i}^{\underline{a}}=\aleph_{i} .
$$

Thus

$$
\left(L, \delta_{i}^{a}+1\right) \models \lambda=\aleph_{1} \& \alpha_{1} \geq \aleph_{2} \& \cdots \& \alpha_{i-1} \geq \aleph_{i} \& \delta_{i}^{\frac{a}{i}} \geq \aleph_{i+1} .
$$

Thus $[\lambda, \lambda+\alpha)$ contains a local $\aleph_{i+1}$.

$(\Rightarrow)$. Suppose that $[\lambda, \lambda+\alpha)$ contains a local $\kappa_{i+1}$. Thus there are $\alpha_{1}, \ldots, \alpha_{i} \in$ $[\lambda, \lambda+\alpha)$ such that

$$
L_{\alpha_{i}+1} \vDash \lambda=\aleph_{1} \& \alpha_{1}=\aleph_{2} \& \cdots \& \alpha_{i}=\kappa_{i+1} .
$$

Clearly $\lambda+\alpha_{1}=\alpha_{1}$. We construct an $I_{\lambda}$-exact pair $(\underline{b}, \underline{c})$ such that $\lambda_{i}^{(\underline{b} \vee \underline{c})} \leq \mu_{\lambda}$. By the induction hypothesis, $\lambda_{i}^{(\underline{b} \vee c)}=\operatorname{dom}^{*}\left(d_{i}^{(b \vee c)}\right)$. So this suffices. We construct $B$ and $C \in 2^{\omega}$ such that $\underline{b}=\operatorname{deg}(B)$ and $\underline{c}=\operatorname{deg}(C)$. Let conditions be as in the proof of Lemmas 1 and 2 under Cases 1 and 2. Let the forcing language $L$ be $L_{\alpha_{i}+1}[\underline{B}, \underline{C}]$. Let $\left\langle\left(P_{j}, Q_{j}\right)\right\rangle_{j \in \omega}$ be a sequence of conditions such that

$P_{0}=Q_{0}=\mathrm{id}$

$\left(P_{2 i+1}, Q_{2 i+1}\right)$ extends $\left(P_{2 i}, Q_{2 i}\right)$ and decides $\varnothing_{i}$;

$\left(P_{2 i+2}, Q_{2 i+2}\right)=\left(P_{2 i+1} * A_{i}, Q_{2 i+1} * A_{i}\right)$

where $\left\langle\varnothing_{i}\right\rangle_{i \in \omega}$ and $\left\langle A_{i}\right\rangle_{i \in \omega}$ are enumerations of the sentences of $\boldsymbol{L}$ and of $\boldsymbol{L}_{\lambda} \cap 2^{\omega}$ respectively. Let $(B, C)=\bigcap_{j}\left[P_{j}, Q_{j}\right] . \lambda \leq \omega_{1}{ }^{(B \oplus C)}$. By the standard argument, all cardinals of $L_{\alpha_{i}+1}$ except for $\aleph_{1}$ are preserved.

$$
L_{\alpha_{i}+1}[B \oplus C] \models \alpha_{1}=\kappa_{1} \& \cdots \& \alpha_{i}=\kappa_{i} .
$$

Thus $\alpha_{i} \geq \delta_{i}^{(\underline{b} \vee \underline{c})}$. So $\lambda_{i}^{(\underline{b} \vee \underline{c})} \leq \alpha_{1}<\alpha \leq \omega \alpha \leq \mu_{\lambda}$. Thus Lemma 4 is proved for this choice of $i$.

We now prove that $\operatorname{dom}^{*}\left(d_{i+1}\right)=\lambda_{i+1}$. If $\lambda<\lambda_{i+1}, \lambda$ does not start an $L$-gap containing a local $\kappa_{i+1}$. By Lemma 4 , for any $\underline{a}$ which is $I_{\lambda}$-exact, $\mu_{\lambda} \in \operatorname{dom} *\left(d_{i}^{a}\right)$. Thus $d_{i+1}(\lambda)$ is defined. $\lambda_{i+1}$ starts an $L$-gap containing a local $\aleph_{i+1}$. By Lemma 4 , $d_{i+1}\left(\lambda_{i+1}\right)$ is undefined.

Finally, suppose $\xi=\omega$. If $\lambda<\lambda_{\omega}$, for some $i \in \omega, \lambda$ does not start an $L$-gap containing a local $\aleph_{i+1}$. By Lemma 4 , for any $I_{\lambda}$-exact $\underline{a}, \mu_{\lambda} \in \operatorname{dom}^{*}\left(d_{i}^{\underline{a}}\right)$. Thus $d_{\omega}(\lambda)$ is defined. $\lambda_{\omega}$ starts an $L$-gap containing a local $\kappa_{i+1}$ for every $i \in \omega$. Thus for any $i \in \omega$ there is an $I_{\lambda_{\omega}}$-exact $\underline{a}$ such that $\mu_{\lambda_{\omega}} \notin \operatorname{dom}^{*}\left(d_{i}^{\underline{a}}\right)$. Thus $d_{\omega}\left(\lambda_{\omega}\right)$ is undefined.

Proof of TheORem 5. Let $\left\langle\alpha_{i}\right\rangle_{i \in \omega}$ be such that for all $i \in \omega,\left(L, \delta_{<\omega}\right) \models \alpha_{i}=\kappa_{i+1}$. It suffices to construct $(\underline{b}, \underline{c}) I_{\lambda_{\omega}}$-exact such that $\alpha_{1}=\lambda_{\omega}^{(b \vee c)}$. Because $\mu_{\lambda_{\omega}}>\omega \alpha_{1}=$ $\alpha_{1}$, by Theorem 4, $\mu_{\lambda_{\omega}} \notin \operatorname{dom}^{*}\left(d_{\omega}^{(b \bigvee c)}\right)$. Thus $d_{\omega+1}\left(\lambda_{\omega}\right)$ is undefined. As in the proof of Lemma $4(\Rightarrow)$, we construct $(\underline{b}, \underline{c})$ such that for all $i \in \omega,\left(L^{(b \vee c)}, \delta_{<\omega}\right) \vDash \alpha_{i}=\aleph_{i}$. Thus $\delta_{<\omega}=\delta_{<\omega}^{(b \vee c)}$. So $\alpha_{1}=\lambda_{\omega}^{(b \vee \varepsilon)}$. 
Let $A_{n}$, for $n<\omega$, be $n$ th-order number theory, i.e. Peano's axioms set in an $n$th order language, where variables of order $i, 1 \leq i \leq n$, range over sets of type $i-1$. Let $A_{\omega}=\bigcup_{n} A_{n}$, in the language with variables of all finite orders. $A_{2}$ is analysis. For $\xi \leq \omega$, we imitate the construction of the ramified analytical hierarchy. A structure for $A_{\xi}$ has the form $\left\langle\left\langle U_{i}\right\rangle_{1 \leq i<\xi} ;+, \cdot, S ; 0\right\rangle$ where $U_{0}=\omega$ and $U_{j+1} \subseteq$ $P\left(U_{j}\right)$; variables of order $j+1$ range over $U_{j}$. Let $M_{\xi}^{\xi}$ be the structure for $A_{\xi}$ with all $U_{j}$ 's, for $j \neq 0$, empty. Form the transfinite sequence $\left\langle M_{\eta}^{\xi}\right\rangle_{\eta}$ by iterating closure under definability in the language of $A_{\xi}$. This hierarchy stops. Let the final structure be $M^{\xi}$ with domains $\left\langle U_{i}^{\xi}\right\rangle_{1 \leq i<n}$; let the closure ordinal be $\gamma_{\xi}$. Then $M^{\xi} \vDash A_{\xi}$. Let $A_{\xi}^{*}$ be $A_{\xi}$ translated into the language of set theory. $L_{\delta_{n}} \cap P^{n}(\omega)$ and $L_{\delta_{<\omega}} \cap P^{n}(\omega)$ are, respectively, the minimal models for $A_{n+1}^{*}$ and $A_{\omega}^{*}$ in which wellfoundedness is absolute. Thus $U_{i+1}^{\dot{n}}=\left(L, \delta_{n}\right) \cap P^{i}(\omega) ; U_{i}^{\omega}=\left(L, \delta_{<\omega}\right) \cap P^{i}(\omega)$ where $U_{i}^{n+1}, U_{i}^{\omega}$ are from $M^{n+1}, M^{\omega}$ respectively. Thus for any $n<\omega, \gamma_{n}=\delta_{n-1}$; furthermore, $\gamma_{\omega}=\delta_{<\omega}$. Let $I^{\xi}=\lambda_{\xi}$ for $\xi \leq \omega$. By Theorem 4, $\bigcup I_{\xi} \cap 2^{\omega}=U_{1}^{\xi}$. Thus $d_{\xi}$ classifies the degrees of reals in $M^{\xi}$.

§4. Comparison with the single jump operation. How similar is the single jump operation $\underline{a} \mapsto \underline{a}^{\prime}$ to an arbitrary operation of the form $\underline{a} \mapsto \underline{a}^{(\xi)}$ for $\xi<\left(\boldsymbol{\kappa}_{1}\right)^{L \underline{a}}$ ? In this section we examine an analogy and a striking disanalogy. As usual, all results are stated for $\underline{a}=0$, but easily generalize to arbitrary $\underline{a}$.

The analogy: Friedberg's completeness theorem $\left(\left(\forall \underline{a} \geq \underline{0}^{\prime}\right)(\exists \underline{c})\left(\underline{a}=\underline{c}^{\prime}\right)\right)$ generalizes to arbitrary transfinite jumps:

THEOREM 6. For any $\xi<\left(\kappa_{1}\right)^{L}$ and any $\underline{a}$, if $\underline{a} \geq \underline{0}^{(\xi)}$ then there is a $\underline{c}$ such that $\underline{a}$ $=\underline{c}^{(\xi)}$.

The disanalogy: the trivial fact that $\underline{0}^{\prime} \leq \underline{a}^{\prime}$ does not generalize in the most straightforward way.

THEOREM 7. For any $\xi<\left(\kappa_{1}\right)^{L}$ and any $\underline{a}, \underline{0}^{(\xi)} \leq \underline{a}^{(\operatorname{Ind}(\xi))}$.

THEOREM 8. For any $\xi<\left(\aleph_{1}\right)^{L}$ there is a $\underline{b}$ such that $\underline{0}^{(\xi)}=\underline{b}^{(\operatorname{Ind}(\xi))}$.

Before we present proofs, notice that if $\xi+\eta<\left(\kappa_{1}\right)^{L}, \underline{0}^{(\xi)(\eta)}=\underline{0}^{(\xi+\eta)}$. This follows by an easy induction on $\eta$.

Proof of TheOrem 6. Suppose $\underline{a} \geq \underline{0}^{(\xi)}$. If $\xi=\alpha+1$, by the relativization of Friedberg's theorem to $\underline{0}^{(\alpha)}$, there is a $\underline{d} \geq \underline{0}^{(\alpha)}, \underline{d}^{\prime}=\underline{a}$. By the induction hypothesis on $\alpha$, there is a $\underline{c}$ such that $\underline{d}=\underline{c}^{(\alpha)}$. Thus $\underline{d}^{\prime}=\underline{c}^{(\alpha+1)}=\underline{a}$.

Suppose that $\xi$ is a limit ordinal. By analogy with Friedberg's argument, we construct a $\underline{c}$ such that $\underline{c}^{(\xi)} \leq \underline{a} \leq \underline{c} \vee \underline{0}^{(\xi)} \leq \underline{c}^{(\xi)}$. Clearly such a $\underline{c}$ is as desired. Suppose $\operatorname{Ind}(\xi)=\omega \beta+n$ and $A \in \underline{a}$. Let the forcing language $\boldsymbol{L}$ be $L_{\beta}[\underline{C}]$. Let $\left\langle\phi_{i}\right\rangle_{i \in \omega}$ be a $\Delta_{n+1}\left(L_{\beta}\right)$ enumeration of the $\Sigma_{n} \cup \Pi_{n}$ sentences of $\boldsymbol{L}$. We shall force with Cohen condition, viewed as finite strings of 0 and 1 . There is a sequence of Cohen conditions $\left\langle\sigma_{i}\right\rangle_{i \in \omega} \in \Delta_{n+1}\left(L_{\beta}\right)$ such that for every $i$ :

$\sigma_{i+1}$ extends $\sigma_{i}$

$\sigma_{2 i}$ decides $\phi_{i}$

$\sigma_{2 i+1}=\sigma_{2 i} A(i)$.

Let $C=\lim _{i \rightarrow \omega} \sigma_{i}$; let $\underline{c}=\operatorname{deg}(C)$. For any $E_{\beta},\left\langle\sigma_{i}\right\rangle_{i \in \omega}$ is coded by a single real recursive in $\operatorname{Th}_{n}\left(E_{\beta}\right) \oplus A$. Select $E_{\beta}$ such that $\operatorname{Th}_{n}\left(E_{\beta}\right) \in \underline{0}^{(\xi)}$. Because $\operatorname{Th}_{n}\left(E_{\beta}\right) \leq_{\mathrm{T}} A$, $C \leq_{\mathrm{T}} A$. Because the even steps are determined only by $\operatorname{Th}_{n}\left(E_{\beta}\right)$ and $A$ is coded into $C$ at the odd steps, $A \leq{ }_{\mathrm{T}} C \oplus \mathrm{Th}_{n}\left(E_{\beta}\right)$. Thus $\underline{c}^{(\xi)} \leq \underline{a} \leq \underline{c} \vee \underline{0}^{(\xi)}$. Finally 
because all conditions belong to $L_{0}$, for any $\eta \leq \beta$ and any $m, L_{\eta} \vDash \Delta_{m}$ CA iff $L_{\eta}[C] \models \Delta_{m}$ CA. Thus for any $\eta \leq \xi, \underline{0}^{(\eta)} \leq \underline{c}^{(\eta)}$. Therefore $\underline{c} \vee \underline{0}^{(\xi)} \leq \underline{c}^{(\xi)}$. Q.E.D.

Proof OF TheOrem 7. Suppose $\xi=\gamma+1$. By induction hypothesis, $\underline{0}^{(\gamma)} \leq \underline{a}^{(\operatorname{Ind}(r))}$. Thus $\underline{0}^{(\gamma+1)} \leq \underline{a}^{(\operatorname{Ind}(\gamma)+1)}$. But $\operatorname{Ind}(\xi)=\operatorname{Ind}(\gamma)+1$. Suppose $\xi$ is a limit. Let $\operatorname{Ind}(\xi)=$ $\omega \beta+n$; let $\operatorname{Ind}^{a}(\operatorname{Ind}(\xi))=\omega \alpha+m \geq \omega \beta+n$. Let $A \in \underline{a}$. By the procedure used in the proof of Lemma 3 , Case 3, in the final paragraph, there is a uniform way of obtaining an $E_{\beta}$ from any $E_{\alpha}[A]$ such that $\mathrm{Th}_{n}\left(E_{\beta}\right) \leq{ }^{\top} \mathrm{Th}_{m}\left(E_{\alpha}[A]\right)$. This suffices.

Proof of Theorem 8. If $\operatorname{Ind}(\xi)=\xi$, Theorem 8 is trivial. So suppose $\operatorname{Ind}(\xi)>$ $\xi$. It suffices to construct a $\underline{b}$ such that $\underline{b}^{(\operatorname{Ind}(\xi))} \leq \underline{0}^{(\xi)}$. Let $\operatorname{Ind}(\xi)=\omega \alpha+n$. Let $\boldsymbol{L}$ be $\boldsymbol{L}_{\alpha}[B]$. Let $\delta$ be the maximum ordinal $\leq \alpha$ which is admissible or a limit of admissibles. We force with modified Steel conditions, with ordinal labels $<\delta$. Let $\left\langle\phi_{i}\right\rangle_{i \in \omega} \in \Delta_{n+1}\left(L_{\alpha}\right)$ enumerate the $\left(\Sigma_{n} \cup \Pi_{n}\right)$ sentences of $\boldsymbol{L}$. Let $\left\langle z_{i}\right\rangle_{i \in \omega} \in \Delta_{n+1}\left(L_{\alpha}\right)$ be a sequence of modified Steel conditions such that for any $i, z_{i+1}$ extends $z_{i}$ and $z_{i}$ decides $\phi_{i}$. By the standard construction, such a sequence exists. Let $B$ be the extension of $\underline{B}$ determined by this sequence. Let $\underline{b}=\operatorname{deg}(B)$. Because $\left\langle z_{i}\right\rangle_{i \in \omega} \in$ $\Delta_{n+1}\left(L_{\alpha}\right)$, for any $E_{\alpha}$ there is an $E_{\alpha}[B]$ such that $\operatorname{Th}_{n}\left(E_{\alpha}[B]\right) \leq_{\mathrm{T}} \mathrm{Th}_{n}\left(E_{\alpha}\right)$. Select $E_{\alpha}$ such that $\mathrm{Th}_{n}\left(E_{\alpha}\right) \in \underline{0}^{(\xi)}$; let $E_{\alpha}[B]$ be determined by $E_{\alpha}$. As in the proof of Lemmas 1 and 2 under Case $3, B$ is a wellfounded tree of sequence numbers of height $\delta$. Thus $\operatorname{Ind}(\xi)<\omega_{1}^{B}$. Thus $\underline{b}^{(\omega \alpha+n)} \leq \operatorname{deg}\left(\operatorname{Th}_{n}\left(E_{\alpha}[B]\right)\right)$.

We finish this section with an application of Theorem 6 .

Corollary. For any $\lambda,\left\{\underline{d} \mid \underline{d} \geq \underline{0}^{(\lambda)}\right\}=\left\{\underline{a}^{\left(\mu_{\lambda}\right)} \mid \underline{a}\right.$ is $I_{\lambda}$-exact $\}$.

PROOF. Let $(\underline{b}, \underline{c})$ be an $I_{\lambda}$-exact pair such that $(\underline{b} \vee \underline{c})^{\left(\mu_{\lambda}\right)} \geq \underline{0}^{(\lambda)}$. Suppose $\underline{d} \geq \underline{0}^{(\lambda)}$. By Theorem 6 , for some $\underline{d}^{*} \geq(\underline{b} \vee \underline{c})^{(2)},\left(\underline{d}^{*}\right)^{\left(-2+\mu_{\lambda}\right)}=\underline{d}$. By $(J)$ of $[4, \S 2]$, for some $I_{\lambda}$-exact pair $\left(\underline{b}^{*}, \underline{c}^{*}\right),\left(\underline{b}^{*} \vee \underline{c}^{*}\right)^{(2)}=d^{*}$. The other direction is just Lemma 3 .

\section{BIBLIOGRAPHY}

[1] G. Boolos and H. Putnam, Degrees of unsolvability of constructible sets of integers, this JourNaL, vol. 33 (1968), pp. 497-513.

[2] R. Boyd, G. HeNSEL and H. PUTNAM, A recursion-theoretic characterization of the ramified analytical hierarchy, Transactions of the American Mathematical Society, vol. 141 (1969), pp. 47-62.

[3] P. COHEN, Set theory and the continuum hypothesis, Benjamin, New York, 1966.

[4] H. HoDEs, Uniform upper bounds on ideals of Turing degrees, this JOURNAL, vol. 43 (1978), pp. 601-612.

[5] R. JENSEN, The fine structure of the constructible universe, Annals of Mathematical Logic, vol. 8 (1972), pp. 1-32.

[6] C. JockusCh and S. Simpson, A degree-theoretic characterization of the ramified analytical hierarchy, Annals of Mathematical Logic, vol. 10 (1976).

[7] S. LeEdS and H. PUTNAM, An intrinsic characterization of the hierarchy of the constructible sets of integers, Logic Colloquium '69, North-Holland, Amsterdam and London, 1971.

[8] W. MARAK and M. SREBenY, Gaps in the constructible universe, Annals of Mathematical Logic, vol. 6 (1974), pp. 359-394.

[9] G. SACKs, Forcing with perfect closed sets, Proceedings of Symposia in Pure Mathematics, vol. 13, American Mathematical Society, Providence, R. I., 1971.

[10] J. STEeL, Ph. D. Thesis, University of California, Berkeley, 1977.

CORNELL UNIVERSITY

ITHACA, NEW YORK 14853 